\title{
A thin-walled beam element based on semi-analytical solution modes
}

\author{
Hansen, Anders Bau; Jönsson, Jeppe
}

Published in:

Thin-Walled Structures

Link to article, DOI:

10.1016/j.tws.2019.106344

Publication date:

2019

Document Version

Peer reviewed version

Link back to DTU Orbit

Citation (APA):

Hansen, A. B., \& Jönsson, J. (2019). A thin-walled beam element based on semi-analytical solution modes.

Thin-Walled Structures, 144, [106344]. https://doi.org/10.1016/j.tws.2019.106344

\section{General rights}

Copyright and moral rights for the publications made accessible in the public portal are retained by the authors and/or other copyright owners and it is a condition of accessing publications that users recognise and abide by the legal requirements associated with these rights.

- Users may download and print one copy of any publication from the public portal for the purpose of private study or research.

- You may not further distribute the material or use it for any profit-making activity or commercial gain

- You may freely distribute the URL identifying the publication in the public portal

If you believe that this document breaches copyright please contact us providing details, and we will remove access to the work immediately and investigate your claim 


\title{
A Thin-Walled Beam Element Based on Semi-Analytical Solution Modes
}

\author{
Anders Bau Hansen ${ }^{\mathrm{a}, \mathrm{b}}$, Jeppe Jönsson ${ }^{\mathrm{b}, *}$ \\ ${ }^{a}$ NIRAS A/S, Sortemosevej 19, DK-3450 Allerød, Denmark \\ ${ }^{b}$ Technical University of Denmark, Department of Civil Engineering, Brovej Building 118, DK-2800 Kgs. Lyngby, Denmark
}

\begin{abstract}
Using energy principles, a thin-walled beam element is introduced for the analysis of beams with deformable crosssections that are prone to distortion. The beam element is based on previously attained semi-analytical displacement solution modes of an advanced thin-walled beam model. The first-order beam element for linear analysis handles shear deformations related to both Timoshenko and Mindlin-Reissner type deformations, warping effects of torsion, crosssection distortion including associated warping effects, as well as the transverse displacement effect from normal stress. The formulation can handle both open and closed cross-sections without special attention. The formulation of the displacement solution modes and the stiffness integration of the products of the advanced displacement modes using the Hadamard product are described. The paper also presents the transformations between modal degrees of freedom and element displacement degrees of freedom. Four examples show the beam element capabilities and good agreement with results obtained using the shell and solid elements of a commercial finite element program. The kinematic assumptions that the thin-walled beam model accommodates leads to local shear stress transfer at corners. This transfer of shear stresses is not normally seen in thin-walled beam formulations or shell models. However, the shear transfer is verified through examination of a finite element model using solid elements.
\end{abstract}

Keywords: Thin-walled beams, beam element, distortional beam theory, shear deformations, corner shear

\section{Introduction}

Since the industrialisation of steel production in the late nineteenth-century thin-walled metal members have been utilised in structures such as bridges, buildings, aeroplanes and ships, see [1]. The main reason for this is the high stiffness-to-weight ratio of the thin-walled members. This led to the development of theories enabling analysis and the assessment of such members since it was realised that during flexure and torsion, the beams generated nonnegligible normal stresses due to warping displacements of the cross-section. Vlasov introduced the well-known one-dimensional thin-walled beam theory, [2]. With this theory, the torsional warping effects of open thin-walled beams were included. Kollbrunner \& Hajdin [3] expanded the theory to include cross-sections with closed cells. In line with the development of the finite element method, thin-walled beam elements were introduced having a total of 14 degrees of freedom, see for example [4, 5]. The inclusion of extra degrees of freedom was to handle the torque and bimoment acting upon the member. However, such finite beam elements do not include the distortional effects of the cross-section. Consequently, a thin-walled beam formulation incorporating cross-sectional distortion is the Generalised Beam Theory (GBT), which was introduced

\footnotetext{
* Corresponding author

Email address: jej@byg.dtu.dk (Jeppe Jönsson)
}

by Schardt in 1966 under the name "Verallgemeinerte Technische Biegetheorie" (VTB) [6, 7]. Kollbrunner and Hajdin describe a similar approach to the introduction of a consistent distortional beam theory in [8]. Nonetheless, with the research performed by Davies and co-workers, GBT was spread outside the German-speaking academic society 9, 10. Since then, different academic groups have contributed to its development - e.g. Simão \& da Silva [11, the group around Camotim [12, 13, 14, 15, 16, 17, or by Ranzi and his colleges [18, 19] just to mention some of the many contributors. The general idea of GBT is to represent and discretise the cross-section along the wall centre line to find distortional transverse displacement modes that have orthogonal warping modes. The approximate displacement modes are achieved solving specific eigenvalue problems related to the beam equilibrium equations. Besides, each mode is affiliated to an axial amplitude function. Therefore, considering standard beam finite element formulations, the axial variations affiliated to each crosssectional displacement field is assumed to be Hermite cubic polynomials.

Alongside the development of GBT, other thin-walled beam analysis methods were developed too. One of these methods is the Finite Strip Method (FSM), Cheung [20]. Several versions of the original FSM formulation have been presented, for example the constrained Finite Strip Method (cFSM), by Ádány \& Schafer [21, 22, 23]. The constraints introduced, enables a subdivision of the displacements into 
specific displacement modes, which allows a modal decomposition as in GBT. Both Ádány et al. 24 and Silvestre et al. [15] give detailed comparisons of cFSM and GBT. The approach to Generalised Eigenvectors (GE), presented by Genoese and co-workers 25, 26, was recently compared to the GBT method by Garcea et al. [27. The GE method is very versatile due to the discretisation of the cross-section using finite shell elements. On the other hand, this discretisation increases the number of elements compared to the method presented in this paper as well as the GBT method. Besides, the GE method is oriented towards anisotropic members, whereas the present theory concerns isotropic material behaviour.

In the last decade, semi-analytical methods, which are closely related to GBT, have been presented. In these methods, the cross-section is discretised into wall elements with local displacement interpolation. The beam solution modes are found through an analytic solution of the equilibrium equations related to the cross-sections. Among others, this has been done by Jönsson \& Andreassen [28], and Vieira et al. [29, 30] who adapt the strong analytical approach of solving the beam differential equations. Solutions to the coupled beam equilibrium equations are deduced through the solution of a related quadratic eigenvalue problem. Both Jönsson \& Andreassen [28] and Vieira et al. [29, 30] introduce a wall element with nodal degrees of freedom and displacements being interpolated by use of standard "beam" type shape functions when they discretise the cross-section. Hence, the cross-sectional displacement fields are directly found as eigenvectors. Vieira et al. 29, 30 use a spectral transformation to transform the complex eigenvectors, whereas Jönsson \& Andreassen [28] as well as the method presented in this paper, directly use the complex solution vectors with the associated complex attenuation functions as beam displacement modes.

The present paper presents a novel formulation of a beam element based on the semi-analytical thin-walled beam theory introduced by the authors in [31. The fundamental modes deduced in this paper are not identical, but similar to the semi-analytical beam modes presented by Vieira 32, they are, however, derived using a different orthogonalisation approach. Using the theory proposed by the authors, a coupled system of beam differential equilibrium equations is derived by taking variations in the strain energy. The theory approximates the crosssectional displacement field by use of discrete wall elements and uses exact analytical solution functions to express the axial variation along the beam, which are deduced from the related differential equilibrium equations. The solution of the differential equations involves a decoupling that leads to cross-sectional displacement fields with associated solution functions that correspond to the amplitude functions. The essential idea of this beam model is that the formulation is based on approximated cross-sectional displacement fields that have associated axial amplitude functions, which are the exact analytical solutions of the homogeneous beam differential equations. While knowing the cross-sectional displacement fields and their associated amplitude functions, it is possible to formulate a beam element adopting these exact solution modes as interpolation functions. In fact, with a linear combination of these preestablished displacement modes, it is possible to develop a beam element. The main and very important feature of the presented method, when compared to GBT, is that GBT uses Hermite polynomial functions to describe the axial variation, whereas the present theory uses the exact solution functions and therefore a further discretisation with multiple elements along the beam axis is not necessary.

Another essential feature of the beam model presented here, is the use of generic wall elements having six degrees of freedom at each node. Consequently, it is possible to connect the beam end cross-section to other finite elements, e.g. finite shell elements. Accordingly, the wall elements used to discretise the cross-section are discrete straight wall elements. Both displacement degrees of freedom, as well as rotational degrees of freedom, are taken into account. Therefore, even with a coarse mesh both global and local distortional modes become a part of the solution space - a space containing cross-sectional displacement fields that are found as solutions to the equilibrium equations. These solutions are found through the solution of the related polynomial eigenvalue problem. From the eigenvalue problem, eigenvectors are extracted as cross-sectional displacement fields with associated exact axial amplitude functions depending on the eigenvalues. In combination, a displacement field and an axial amplitude function describe a beam displacement mode. The modes are grouped into two families: those having eigenvalues equal zero, and those having eigenvalues different from zero. The former include global modes with polynomial amplitudes of maximum third-order reflecting the twelve fundamental beam displacement fields. In the latter case, beam displacement modes with amplitudes having exponential decays are considered. In these cases, the eigenvalues represent an inverse length scale parameter related to the St. Venant principle through an axial decay effect.

\section{The beam model}

An arbitrary thin-walled beam element is located in a global Cartesian coordinate system spanned by the axes $(X, Y, Z)$. The beam axis is assumed to be straight and parallel to the $Z$-axis with the cross-sectional plane being orthogonal to this, as illustrated in Figure 1. Furthermore, a local, right-handed orthogonal coordinate system is introduced in the cross-section with $(n, s, z)$ as the normal, tangential and axial directions.

\subsection{Kinematics}

The displacement of a material point within a beam element is given as a sum of cross-sectional displacement fields multiplied by amplitude functions, which vary along 


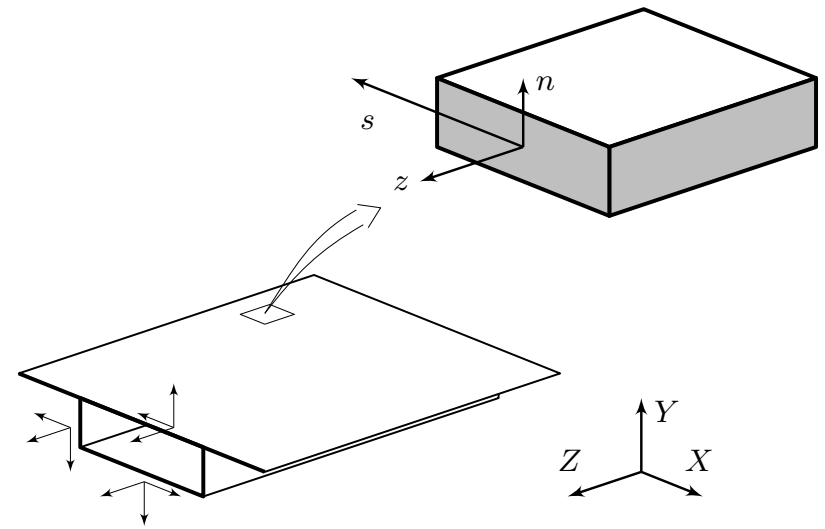

Figure 1: Global and local Cartesian coordinate systems regarding a thin-walled beam

the longitudinal beam axis. The intensities of the amplitude functions are determined by the constants of integration, which become the modal degrees of freedom of the beam model. In other words, the displacements are formulated in the three local coordinate directions $u_{n}, u_{s}$ and $u_{z}$ as a sum of $m$ displacement fields where each displacement field is referred to by index $i$. Hence, the three displacements are given as:

$$
\begin{aligned}
u_{n}(s, z) & =\sum_{i=1}^{m} w_{n}^{i}(s) \psi_{i}(z) c_{i} \\
u_{s}(n, s, z) & =\sum_{i=1}^{m}\left[w_{s}^{i}(s)-n w_{n, s}^{i}(s)\right] \psi_{i}(z) c_{i} \\
u_{z}(n, s, z) & =\sum_{i=1}^{m}\left[\Omega^{i}(s)+n \alpha^{i}(s)\right] \eta_{i}(z) c_{i}
\end{aligned}
$$

where $w_{n}^{i}(s), w_{s}^{i}(s), \Omega^{i}(s)$ and $\alpha^{i}(s)$ are displacement components of the interpolated cross-section displacement mode $i$ with reference to the mid-line of the wall elements. The displacement formulation at hand uses a Kirchhoff like displacement behaviour in the $n, s$-plane, a MindlinReissner like shear behaviour through the wall-thickness in the $n, z$-plane combined with a Timoshenko like shear behaviour in the $s, z$-plane. For illustration purpose, Figure 2 illustrates the meaning of the different displacement components. Derivatives with respect to $n$ - and $s$-coordinates are given as subscripts following a comma whereas axial derivatives are denoted by a prime. The axial amplitude functions that describe the axial variation of a given crosssection displacement field are denoted $\psi_{i}(z)$ and $\eta_{i}(z)$. The transverse displacements of a cross-section are associated with the amplitude function $\psi(z)$, whereas the amplitude function $\eta(z)$ is related to the warping displacements, which are displacements that are orthogonal to the cross-sectional plane. This distinction between amplitude functions has been chosen in order to keep a clearer relation of the kinematics in Equation (1) to the kinematics of conventional beam theories. Therefore, the two independent amplitude functions are used. Nonetheless, later, during the solution of the differential equilibrium equations, the assumption: $\psi_{i}(z)=\eta_{i}(z)$ from 31 is adopted. A comment to the deformation formulations in Equation (1) is that the intensity of each mode $i$ is controlled by the constant $c_{i}$. These constants correspond to the modal degrees of freedom and reflect the intensities of the amplitude functions belonging to each displacement mode in the summation of modal displacements in Equation (1).

In order to determine natural cross-sectional displacement fields, this theory discretises the cross-section into straight wall elements. Each wall element has two nodes with three translational degrees of freedom and three rotational degrees of freedom. Accordingly, the twelve nodal degrees of freedom related to a wall element are collected in the column vectors $\mathbf{v}_{w}^{e l}$ and $\mathbf{v}_{\Omega}^{e l^{i}}$, respectively, with six degrees of freedom in $\mathbf{v}_{w}^{e l}{ }^{i}$ related to translational deformations whereas the remaining six degrees of freedom are collected in $\mathbf{v}_{\Omega}^{e l}$ since they are related to warping deformations. Here and in the following, vectors and matrices will be denoted by non-slanted, boldfaced, letters. The wall element deformations are determined by use of linear interpolation functions given in the row interpolation vectors $\mathbf{N}_{s}(s)$ and $\mathbf{N}_{\alpha}(s)$, and also cubic interpolation functions given in the row vectors $\mathbf{N}_{n}(s)$ and $\mathbf{N}_{\Omega}(s)$ (see also [33]). Now, considering a cross-sectional displacement field $i$, the displacement vector components of a wall element may be written in terms of interpolation vectors and nodal degrees of freedom vectors as follows:

$$
\begin{aligned}
w_{s}^{i}(s)=\mathbf{N}_{s}(s) \mathbf{v}_{w}^{e l^{i}}, & \alpha^{i}(s)=\mathbf{N}_{\alpha}(s) \mathbf{v}_{\Omega}^{e l^{i}} \\
w_{n}^{i}(s)=\mathbf{N}_{n}(s) \mathbf{v}_{w}^{e l^{i}}, & \Omega^{i}(s)=\mathbf{N}_{\Omega}(s) \mathbf{v}_{\Omega}^{e l^{i}}
\end{aligned}
$$

Due to the mode formulation, it is convenient to introduce two wall element deformation vectors $\mathbf{u}_{w}^{e l}(z)$ and $\mathbf{u}_{\Omega}^{e l}(z)$ as the sum of all $m$ displacement modes including the nodal degrees of freedom vectors, the axial amplitude functions, and the constants controlling the mode intensities. Hence, the deformation vectors of a single wall element are computed as the sum of $m$ displacement modes as follows:

$$
\begin{aligned}
& \mathbf{u}_{w}^{e l}(z)=\sum_{i=1}^{m} \mathbf{v}_{w}^{e l^{i}} \psi_{i}(z) c_{i} \\
& \mathbf{u}_{\Omega}^{e l}(z)=\sum_{i=1}^{m} \mathbf{v}_{\Omega}^{e l} \psi_{i}(z) c_{i}
\end{aligned}
$$

The interpolation vectors in Equation (2) are independent of the specific displacement fields. Therefore, the interpolation vectors are the same for all modes with respect to the same wall element. Consequently, for a single wall element, the displacements in Equation (1) may be inter- 


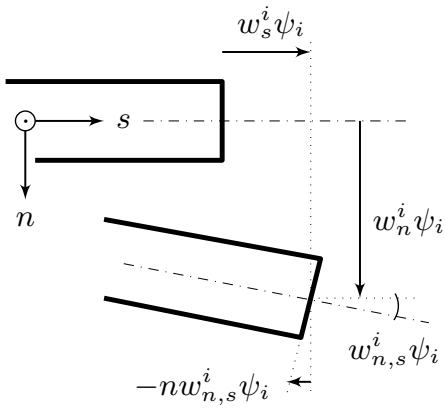

(a) n,s-plane having a Kirchhoff like behaviour

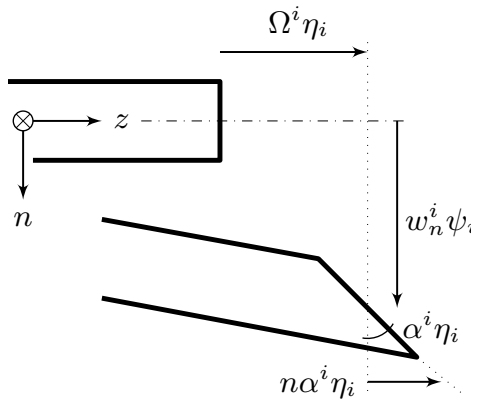

(b) $n, z$-plane having a Mindlin-Reissner like behaviour

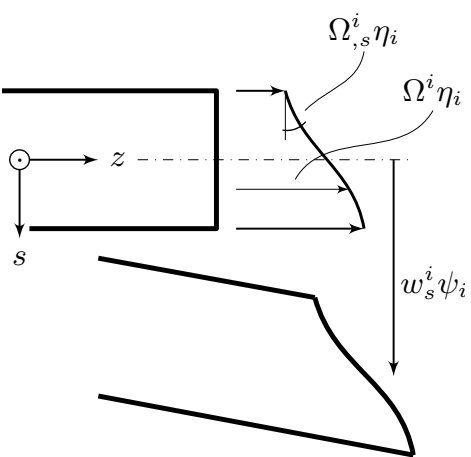

(c) $s, z$-plane having a Timoshenko like behaviour

Figure 2: Allowable deformation patterns regarding a beam element plate for a single mode $i$

polated as:

$$
\begin{aligned}
u_{n}(s, z) & =\mathbf{N}_{n}(s) \mathbf{u}_{w}^{e l}(z) \\
u_{s}(n, s, z) & =\left[\mathbf{N}_{s}(s)-n \mathbf{N}_{n, s}(s)\right] \mathbf{u}_{w}^{e l}(z) \\
u_{z}(n, s, z) & =\left[\mathbf{N}_{\Omega}(s)+n \mathbf{N}_{\alpha}(s)\right] \mathbf{u}_{\Omega}^{e l}(z)
\end{aligned}
$$

in which $\mathbf{u}_{w}^{e l}$ and $\mathbf{u}_{\Omega}^{e l}$ are column vectors containing the nodal deformations related to a single wall element as illustrated in Figure 3 To ease the notation, we will in the following omit the function handle $(z)$ that shows the explicit dependency on the beam axis coordinate, except a few places where there is a specific need for it.

As in [31] the strains are based on the linear small displacement hypothesis and the deformation formulations from Equation (1). This renders the following strain formulations where the normal strain becomes zero $\left(\varepsilon_{n n}=0\right)$, but the transverse distortional normal strain and the axial normal strain are given as:

$$
\varepsilon_{s s}=u_{s, s} \quad \text { and } \quad \varepsilon_{z z}=u_{z}^{\prime}
$$

The non-null engineering shear strains are found as:

$$
\gamma_{s z}=u_{s}^{\prime}+u_{z, s} \quad \text { and } \quad \gamma_{n z}=u_{n}^{\prime}+u_{z, n}
$$

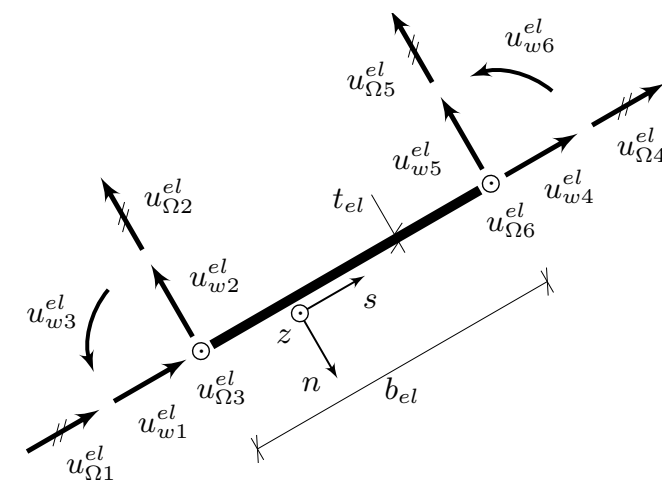

Figure 3: Wall element, which is used to discretise the crosssection also illustrating the deformation components from $\mathbf{u}_{w}^{e l}$ and $\mathbf{u}_{\Omega}^{e l}$, respectively whilst $\gamma_{n s}=0$.

\subsection{Strain energy and cross-sectional stiffness matrices}

Since a linear elastic first-order analysis is considered, the constitutive equations follow from the assumption of zero normal stress $\sigma_{n n}=0$ as given in the following Equation (7). Here, the stresses are directly found from the strains. The material properties are assumed to be elastic and isotropic with a modulus of elasticity $E$, a shear modulus: $G=E /(2(1+\nu))$, and a plate elasticity modulus: $E_{s}=E /\left(1-\nu^{2}\right)$ in which $\nu$ is the Poisson ratio. The non-null stresses are then found using the linear constitutive relations including coupling terms between axial and transverse stresses related to deformations within the cross-sectional plane due to the Poisson effect. Hence,

$$
\left[\begin{array}{c}
\sigma_{s s} \\
\sigma_{z z} \\
\tau_{s z} \\
\tau_{n z}
\end{array}\right]=\left[\begin{array}{cccc}
E_{s} & \nu E_{s} & 0 & 0 \\
\nu E_{s} & E_{s} & 0 & 0 \\
0 & 0 & G & 0 \\
0 & 0 & 0 & G
\end{array}\right]\left[\begin{array}{c}
\varepsilon_{s s} \\
\varepsilon_{z z} \\
\gamma_{s z} \\
\gamma_{n z}
\end{array}\right]
$$

The stresses $\sigma_{s s}, \sigma_{z z}$ and $\tau_{s z}$ depend on all three local coordinates $(n, s, z)$ whereas $\tau_{n z}$ only depends on the $(s, z)$ coordinates, since it is constant through the plate thickness (well-knowing the true variation is parabolic). Figure 4 illustrates the included stresses and their distribution through the thickness. Due to the difference between shear formulations in the two plate directions of a wall element and due to the very limited influence of plate shear, a

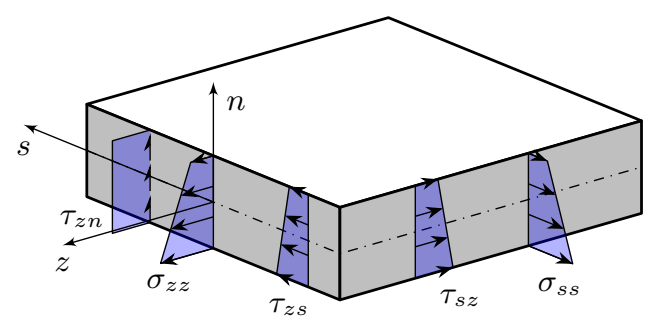

Figure 4: Allowable stress variations at the wall cut-out from Figure 1 with $\sigma_{n n}=0$ and $\tau_{n s}=0$ 
specific shear correction factor has not been implemented. This is done even though Timoshenko 34 already in 1921 introduced a correction factor to adjust the influence of shear. Through time, several considerations have been made on this correction, see for example Cowper [35] or finite element textbooks [36]. However, well-knowing the shear variation through a wall is incorrect, its influence is assumed to be small and a correction would be out of the scope of this paper.

The linear elastic strain energy is formulated by integrating the strain energy density over the full continuum and by substituting stress terms using Equation (7). Accordingly, the linear elastic strain energy becomes:

$$
\begin{aligned}
& U=\frac{1}{2} \int_{V}\left(E_{s} \varepsilon_{s s}^{2}+E_{s} \varepsilon_{z z}^{2}\right.+2 E_{s} \varepsilon_{s s} \varepsilon_{z z} \nu \\
&\left.+G \gamma_{n z}^{2}+G \gamma_{s z}^{2}\right) d V
\end{aligned}
$$

The strain energy is partly integrated by performing integration over the cross-section area. The total strain energy expression is found by adding up the cross-sectional integrations of each wall element, i.e. integrating over both wall width and wall thickness and assembling the crosssectional wall elements into the strain energy using standard finite element methods.

The strain energy is formulated by use of cross-sectional stiffness matrices. Table 1 presents local wall element stiffness matrices deduced by substitution of the strains from Equation (5) and (6) as well as the displacements from Equation (4) into the energy in Equation (8). Accordingly, by considering standard transformations and assembling procedures, global cross-sectional stiffness matrices are derived, see also Cook et al. [36. The assembling procedure leads to the following strain energy formulation:

$$
U=\frac{1}{2} \int_{0}^{\ell}\left[\begin{array}{c}
\mathbf{u}_{w} \\
\mathbf{u}_{\Omega} \\
\mathbf{u}_{w}^{\prime} \\
\mathbf{u}_{\Omega}^{\prime}
\end{array}\right]^{\top}\left[\begin{array}{cccc}
\mathbf{K}_{w w}^{s} & \cdot & \cdot & \mathbf{K}_{w \Omega}^{s \sigma} \\
\cdot & \mathbf{K}_{\Omega \Omega}^{\gamma} & \mathbf{K}_{\Omega w}^{\gamma} & \cdot \\
\cdot & \mathbf{K}_{w \Omega}^{\gamma} & \mathbf{K}_{w w}^{\gamma} & \cdot \\
\mathbf{K}_{\Omega w}^{\sigma s} & \cdot & \cdot & \mathbf{K}_{\Omega \Omega}^{\sigma}
\end{array}\right]\left[\begin{array}{c}
\mathbf{u}_{w} \\
\mathbf{u}_{\Omega} \\
\mathbf{u}_{w}^{\prime} \\
\mathbf{u}_{\Omega}^{\prime}
\end{array}\right] d z
$$

Hence, the cross-sectional deformation vectors $\mathbf{u}_{w}$ and $\mathbf{u}_{\Omega}$ contain all wall element deformation vectors from Equation (3) as a result of the assembling procedure. Additionally, in Equation (9) and throughout the paper a dot [ · ] represents a suitable null matrix. The superscripts $s, \sigma$ and $\gamma$ indicate terms related to transverse, axial or shear stresses and strains, respectively.

It is convenient in the following to introduce a common deformation vector containing both translational and warping deformations:

$$
\mathbf{u}(z)=\left[\begin{array}{l}
\mathbf{u}_{w}(z) \\
\mathbf{u}_{\Omega}(z)
\end{array}\right]
$$

At the same time, the cross-sectional stiffness matrices are
Table 1: Local wall element stiffness matrices

$$
\begin{aligned}
& \mathbf{k}_{w w}^{s}=\int_{0}^{b_{e l}}\left(t_{e l} E_{s} \mathbf{N}_{s, s}^{\top} \mathbf{N}_{s, s}+\frac{t_{e l}^{3}}{12} E_{s} \mathbf{N}_{n, s s}^{\top} \mathbf{N}_{n, s s}\right) d s \\
& \mathbf{k}_{\Omega \Omega}^{\sigma}=\int_{0}^{b_{e l}}\left(t_{e l} E_{s} \mathbf{N}_{\Omega}^{\top} \mathbf{N}_{\Omega}+\frac{t_{e l}^{3}}{12} E_{s} \mathbf{N}_{\alpha}^{\top} \mathbf{N}_{\alpha}\right) d s \\
& \mathbf{k}_{w \Omega}^{s \sigma}=\int_{0}^{b_{e l}}\left(t_{e l} \nu E_{s} \mathbf{N}_{s, s}^{\top} \mathbf{N}_{\Omega}-\frac{t_{e l}^{3}}{12} \nu E_{s} \mathbf{N}_{n, s s}^{\top} \mathbf{N}_{\alpha}\right) d s \\
& \mathbf{k}_{\Omega w}^{\sigma s}=\int_{0}^{b_{e l}}\left(t_{e l} \nu E_{s} \mathbf{N}_{\Omega}^{\top} \mathbf{N}_{s, s}-\frac{t_{e l}^{3}}{12} \nu E_{s} \mathbf{N}_{\alpha}^{\top} \mathbf{N}_{n, s s}\right) d s \\
& \mathbf{k}_{\Omega \Omega}^{\gamma}=\int_{0}^{b_{e l}}\left(t_{e l} G \mathbf{N}_{\alpha}^{\top} \mathbf{N}_{\alpha}+t_{e l} G \mathbf{N}_{\Omega, s}^{\top} \mathbf{N}_{\Omega, s}+\frac{t_{e l}^{3}}{12} G \mathbf{N}_{\alpha, s}^{\top} \mathbf{N}_{\alpha, s}\right) d s \\
& \mathbf{k}_{w w}^{\gamma}=\int_{0}^{b_{e l}}\left(t_{e l} G \mathbf{N}_{n}^{\top} \mathbf{N}_{n}+t_{e l} G \mathbf{N}_{s}^{\top} \mathbf{N}_{s}+\frac{t_{e l}^{3}}{12} G \mathbf{N}_{n, s}^{\top} \mathbf{N}_{n, s}\right) d s \\
& \mathbf{k}_{\Omega w}^{\gamma}=\int_{0}^{b_{e l}}\left(t_{e l} G \mathbf{N}_{\alpha}^{\top} \mathbf{N}_{n}+t_{e l} G \mathbf{N}_{\Omega, s}^{\top} \mathbf{N}_{s}-\frac{t_{e l}^{3}}{12} G \mathbf{N}_{\alpha, s}^{\top} \mathbf{N}_{n, s}\right) d s \\
& \mathbf{k}_{w \Omega}^{\gamma}=\int_{0}^{b_{e l}}\left(t_{e l} G \mathbf{N}_{n}^{\top} \mathbf{N}_{\alpha}+t_{e l} G \mathbf{N}_{s}^{\top} \mathbf{N}_{\Omega, s}-\frac{t_{e l}^{3}}{12} G \mathbf{N}_{n, s}^{\top} \mathbf{N}_{\alpha, s}\right) d s
\end{aligned}
$$

grouped in blocks as follows:

$$
\begin{aligned}
& \mathbf{K}_{a a}=\left[\begin{array}{cc}
\mathbf{K}_{w w}^{s} & \cdot \\
\cdot & \mathbf{K}_{\Omega \Omega}^{\gamma}
\end{array}\right], \mathbf{K}_{a b}=\left[\begin{array}{cc}
\cdot & \mathbf{K}_{w \Omega}^{s \sigma} \\
\mathbf{K}_{\Omega w}^{\gamma} & \cdot
\end{array}\right], \\
& \mathbf{K}_{b a}=\mathbf{K}_{a b}^{\top} \text { and } \mathbf{K}_{b b}=\left[\begin{array}{cc}
\mathbf{K}_{w w}^{\gamma} & \cdot \\
\cdot & \mathbf{K}_{\Omega \Omega}^{\sigma}
\end{array}\right]
\end{aligned}
$$

in which each block matrix is $n \times n$, where $n$ is six times the number of nodes used to discretise the cross-section. Thus, with this block notation, the strain energy from Equation (9) is written as:

$$
U=\frac{1}{2} \int_{0}^{\ell}\left[\begin{array}{l}
\mathbf{u} \\
\mathbf{u}^{\prime}
\end{array}\right]^{\top}\left[\begin{array}{ll}
\mathbf{K}_{a a} & \mathbf{K}_{a b} \\
\mathbf{K}_{b a} & \mathbf{K}_{b b}
\end{array}\right]\left[\begin{array}{l}
\mathbf{u} \\
\mathbf{u}^{\prime}
\end{array}\right] d z
$$

\subsection{Modal displacement field and solution space}

From the strain energy, in Equation (12), beam equilibrium equations are deduced using variational principles, i.e. virtual work principle and partial integration as in [31]. This leads to:

$$
\mathbf{K}_{2} \mathbf{u}^{\prime \prime}+\mathbf{K}_{1} \mathbf{u}^{\prime}+\mathbf{K}_{0} \mathbf{u}=\mathbf{0}
$$

where the stiffness matrices $\mathbf{K}_{0}, \mathbf{K}_{1}$ and $\mathbf{K}_{2}$ are defined as blocks of cross-sectional stiffness matrices as follows:

$$
\begin{aligned}
& \mathbf{K}_{0}=\left[\begin{array}{cc}
\mathbf{K}_{w w}^{s} & \cdot \\
\cdot & -\mathbf{K}_{\Omega \Omega}^{\gamma}
\end{array}\right], \\
& \mathbf{K}_{1}=\left[\begin{array}{cc}
\cdot & \mathbf{K}_{w \Omega}^{s \sigma}-\mathbf{K}_{w \Omega}^{\gamma} \\
\mathbf{K}_{\Omega w}^{\sigma s}-\mathbf{K}_{\Omega w}^{\gamma} & .
\end{array}\right] \text {, } \\
& \mathbf{K}_{2}=\left[\begin{array}{cc}
-\mathbf{K}_{w w}^{\gamma} & \cdot \\
\cdot & \mathbf{K}_{\Omega \Omega}^{\sigma}
\end{array}\right]
\end{aligned}
$$

The full homogeneous solution space of the second-order differential equation, i.e. Equation (13), contains two parts - one related to fundamental modes having a polynomial 
amplitude function, and the second one related to modes with exponential amplitude functions. Hence, the two solution spaces are found as:

- a set of twelve fundamental displacement modes with polynomial amplitude variations collected in $\mathbf{u}_{p}$. Hence, $\mathbf{u}_{p}$ contains $n_{z}=12$ modes.

- a set of displacement modes having exponential amplitude functions collected in $\mathbf{u}_{e}$. The number of modes in $\mathbf{u}_{e}$ depends on the discretisation of the cross-section such that $\mathbf{u}_{e}$ contains $n_{e}=2 n-n_{z}$ modes. Furthermore, these modes might include pairs of complex conjugated modes.

Let us detail the formulation of these modes in the following.

\subsubsection{Fundamental modes}

The twelve solutions that are categorised as fundamental modes having a polynomial amplitude function are explicitly written as:

$$
\mathbf{u}_{p}(z)=\mathbf{V}_{p} \boldsymbol{\Psi}_{p}(z) \mathbf{T}_{p} \mathbf{c}_{p}
$$

where the cross-sectional nodal degrees of freedom vectors are collected as columns in four block matrices in $\mathbf{V}_{p}=\left[\begin{array}{llll}\mathbf{V}_{3} & \mathbf{V}_{2} & \mathbf{V}_{1} & \mathbf{V}_{0}\end{array}\right]$. The blocks act as coefficients to the polynomial functions $z^{3}, z^{2}, z$, and 1 , respectively. However, the derivation of these cross-sectional nodal degrees of freedom vectors will not be shown here but can be found in the paper 31. The amplitude matrix, the constant-transformation matrix, and the column vector with the intensity-constants of each mode (modal constants) have the following format:

$$
\mathbf{\Psi}_{p}=\left[\begin{array}{cccc}
\frac{z^{3}}{6} \mathbf{I}_{p} & \cdot & \cdot & \cdot \\
\cdot & \frac{z^{2}}{2} \mathbf{I}_{p} & \cdot & \cdot \\
\cdot & \cdot & z \mathbf{I}_{p} & \cdot \\
\cdot & \cdot & \cdot & \mathbf{I}_{p}
\end{array}\right], \quad \mathbf{T}_{p}=\left[\begin{array}{c}
\mathbf{I}_{p} \\
\mathbf{I}_{p} \\
\mathbf{I}_{p} \\
\mathbf{I}_{p}
\end{array}\right], \quad \mathbf{c}_{p}=\left[\begin{array}{c}
c_{p_{1}} \\
c_{p_{2}} \\
\vdots \\
c_{p_{n_{z}}}
\end{array}\right]
$$

in which $\mathbf{I}_{p}$ is an identity matrix of size $n_{z} \times n_{z}$ that is $12 \times 12$ reflecting the number of fundamental modes.

Now, seeking the first derivative of $\mathbf{u}_{p}(z)$ in Equation (15) is traditionally performed by differentiating the amplitude matrix $\boldsymbol{\Psi}_{p}(z)$, which is dependent on the $z$ variable. However, in this (polynomial) case a state-space vector formulation will be used as in the coming exponential solution formulation. Therefore, it is realised that by keeping the amplitude matrix as it is and instead shifting the nodal degree of freedom block vectors in $\mathbf{V}_{p}$ one place to the right the first derivative of the displacement vector $\mathbf{u}_{p}^{\prime}(z)$ can be written as: $\mathbf{u}_{p}^{\prime}(z)=\dot{\mathbf{u}}_{p}(z)$, where the statespace formulation of the displacement-vector-derivative is given as:

$$
\dot{\mathbf{u}}_{p}(z)=\dot{\mathbf{V}}_{p} \boldsymbol{\Psi}_{p}(z) \mathbf{T}_{p} \mathbf{c}_{p}
$$

In which $\dot{\mathbf{V}}_{p}=\left[\begin{array}{llll}\mathbf{0} & \mathbf{V}_{3} & \mathbf{V}_{2} & \mathbf{V}_{1}\end{array}\right]$ is the result of the differentiation operation of $\mathbf{u}_{p}(z)$. Thus, we have introduced the notation $\dot{\mathbf{u}}_{p}$ in this special formulation of the derivative. With this refinement, the polynomial solution and its first derivative, Equation (15) and (17), is conveniently written in state-space vector format as:

$$
\left[\begin{array}{c}
\mathbf{u}_{p} \\
\dot{\mathbf{u}}_{p}
\end{array}\right]=\left[\begin{array}{c}
\mathbf{V}_{p} \\
\dot{\mathbf{V}}_{p}
\end{array}\right] \boldsymbol{\Psi}_{p}(z) \mathbf{T}_{p} \mathbf{c}_{p}
$$

where differentiation of the amplitude functions has been avoided by just shifting the mode vectors and keeping the amplitude matrix $\boldsymbol{\Psi}_{p}$.

\subsubsection{Exponential modes}

In performing the task of solving the second-order differential equation system, it is common to rewrite the equations into a first-order equation system by introducing a state-vector using a special notation for the derivative of a vector, see for example Tisseur and Meerbergen in [37. However, this correctly and as mathematically expected expands the number of solutions and the size of the problem and ruins the conventional notion of orthogonal solution modes. Nonetheless, the variable $\dot{\mathbf{u}}$ defined as the first derivative of the displacement field is introduced. Hence,

$$
\dot{\mathbf{u}}(z) \equiv \mathbf{u}^{\prime}(z)
$$

Following the procedure outlined by Tisseur and Meerbergen [37] by substituting $\dot{\mathbf{u}}(z)$ into Equation 13 for the first derivatives, the second-order differential equation system can be equivalently written as:

$$
\left[\begin{array}{cc}
\mathbf{K}_{0} & \cdot \\
\cdot & -\mathbf{K}_{2}
\end{array}\right]\left[\begin{array}{c}
\mathbf{u} \\
\dot{\mathbf{u}}
\end{array}\right]+\left[\begin{array}{cc}
\mathbf{K}_{1} & \mathbf{K}_{2} \\
\mathbf{K}_{2} & \cdot
\end{array}\right]\left[\begin{array}{l}
\mathbf{u} \\
\dot{\mathbf{u}}
\end{array}\right]^{\prime}=\left[\begin{array}{l}
\mathbf{0} \\
\mathbf{0}
\end{array}\right]
$$

using the state-vector notation. This first-ordered differential equation system is solved as a generalised eigenvalue problem by assuming exponential solutions where each eigenvector corresponds to a vector with the nodal degrees of freedom and its derivative for a single mode, and the corresponding eigenvalue gives the exponential amplitude. Consequently, the exponential solution space $\mathbf{u}_{e}$ and its first derivative $\dot{\mathbf{u}}_{e}$ can be written as:

$$
\left[\begin{array}{c}
\mathbf{u}_{e} \\
\dot{\mathbf{u}}_{e}
\end{array}\right]=\left[\begin{array}{c}
\mathbf{V}_{e} \\
\dot{\mathbf{V}}_{e}
\end{array}\right] \boldsymbol{\Psi}_{e}(z) \mathbf{c}_{e}
$$

in which the nodal degrees of freedom vectors are the eigenvectors given as:

$$
\left[\begin{array}{c}
\mathbf{V}_{e} \\
\dot{\mathbf{V}}_{e}
\end{array}\right]=\left[\begin{array}{cccc}
\mathbf{v}_{1} & \mathbf{v}_{2} & \ldots & \mathbf{v}_{n_{e}} \\
\dot{\mathbf{v}}_{1} & \dot{\mathbf{v}}_{1} & \ldots & \dot{\mathbf{v}}_{n_{e}}
\end{array}\right]
$$

The amplitude matrix and mode intensity constant vector (modal degrees of freedom) in Equation 21 are:

$$
\boldsymbol{\Psi}_{e}=\left[\begin{array}{cccc}
e^{\lambda_{1} z} & & \\
& e^{\lambda_{2} z} & & \\
& \ddots & \\
& & e^{\lambda_{n_{e}} z}
\end{array}\right], \mathbf{c}_{e}=\left[\begin{array}{c}
c_{e_{1}} \\
c_{e_{2}} \\
\vdots \\
c_{e_{n_{e}}}
\end{array}\right]
$$


Here, $\lambda$ represents the non-null eigenvalue of each mode, which may be complex.

\subsubsection{Homogeneous solution space}

The polynomial and exponential solutions are given as Equations (18) and (21), respectively. Thus, the full homogeneous solution can be written as follows:

$$
\begin{aligned}
{\left[\begin{array}{c}
\mathbf{u} \\
\dot{\mathbf{u}}
\end{array}\right] } & =\left[\begin{array}{c}
\mathbf{u}_{p} \\
\dot{\mathbf{u}}_{p}
\end{array}\right]+\left[\begin{array}{c}
\mathbf{u}_{e} \\
\dot{\mathbf{u}}_{e}
\end{array}\right] \\
& =\left[\begin{array}{cc}
\mathbf{V}_{p} & \mathbf{V}_{e} \\
\dot{\mathbf{V}}_{p} & \dot{\mathbf{V}}_{e}
\end{array}\right]\left[\begin{array}{cc}
\mathbf{\Psi}_{p} & \cdot \\
\cdot & \mathbf{\Psi}_{e}
\end{array}\right]\left[\begin{array}{cc}
\mathbf{T}_{p} & \cdot \\
\cdot & \mathbf{I}_{e}
\end{array}\right]\left[\begin{array}{l}
\mathbf{c}_{p} \\
\mathbf{c}_{e}
\end{array}\right] \\
& =\left[\begin{array}{c}
\mathbf{V} \\
\dot{\mathbf{V}}
\end{array}\right] \mathbf{\Psi} \mathbf{T}_{c} \mathbf{c}
\end{aligned}
$$

where $\mathbf{V}$ is the full mode matrix, $\boldsymbol{\Psi}$ is a common amplitude diagonal matrix containing $\boldsymbol{\Psi}_{p}$ and $\boldsymbol{\Psi}_{e}$ from Equation 16 and (23), respectively; and $\mathbf{T}_{c}$ is a constant-transformation matrix containing $\mathbf{T}_{p}$ from Equation (16) and an identity matrix, $\mathbf{I}_{e}$ of size $n_{e} \times n_{e}$. Furthermore $\mathbf{c}$ is a column vector containing all the mode intensity constants $\mathbf{c}_{p}$ and $\mathbf{c}_{e}$ (being modal degrees of freedom).

\section{Beam element formulation}

Having found the displacement modes of the thin-walled beam element in the previous section, based on the chosen discretisation of the cross-section, this section formulates the beam element using these exact displacement modes as axial interpolation functions between beam end crosssections.

The theory developed here takes only nodal forces at boundaries into account, as well as nodal deformations. This has been chosen in order to keep a simple formulation. Nevertheless, distributed loads acting along the beam member axis can be implemented using a similar method as the one reported by Andreassen \& Jönsson [38.

To derive the beam element formulation, the potential energy is formulated as the sum of the strain energy and the potential from applied loads:

$$
V=U+\Pi
$$

The strain energy $U$ is given in Equation 12 and the potential $\Pi$ of external nodal forces at each end of the beam element is given as:

$$
\Pi=-\left[\begin{array}{c}
\mathbf{u}(0) \\
\mathbf{u}(\ell)
\end{array}\right]^{\top}\left[\begin{array}{l}
\mathbf{f}_{0} \\
\mathbf{f}_{\ell}
\end{array}\right]
$$

where $\mathbf{f}_{0}$ and $\mathbf{f}_{\ell}$ are load vectors. Each load vector refers to one of the two beam ends as indicated by the subscripts. Furthermore, a single load vector contains a load parameter for each nodal degree of freedom in the cross-section.

\subsection{Modal beam stiffness matrix}

To formulate a beam element, we must derive its stiffness matrix. To do so, we consider the strain energy from Equation (12).

As interpolation functions, the natural beam displacement modes found as solutions in the previous section, i.e. Equation (24), will be used. Thus, considering the full homogeneous solution space, a formulation of a modal beam stiffness matrix is presented letting the vectors of constants be perceived as the temporary modal degree of freedom vectors. Later, these temporary modal degrees of freedom vectors are transformed into a nodal degree of freedom space containing the classic deformations and rotations at each node. Consequently, we have a beam element with a number of displacement modes that reflect the exact deformation modes derived for this specific beam element. Thus, substituting the homogeneous solution space and its first derivatives from Equation (24) into the elastic strain energy in Equation $\sqrt{12}$ we have:

$$
U=\frac{1}{2} \int_{0}^{\ell} \mathbf{c}^{\top} \mathbf{T}_{c}^{\top} \boldsymbol{\Psi}^{\top}\left[\begin{array}{c}
\mathbf{V} \\
\dot{\mathbf{V}}
\end{array}\right]^{\top}\left[\begin{array}{cc}
\mathbf{K}_{a a} & \mathbf{K}_{a b} \\
\mathbf{K}_{b a} & \mathbf{K}_{b b}
\end{array}\right]\left[\begin{array}{c}
\mathbf{V} \\
\dot{\mathbf{V}}
\end{array}\right] \boldsymbol{\Psi} \mathbf{T}_{c} \mathbf{c} d z
$$

and in integrated form it follows as:

$$
U=\frac{1}{2} \mathbf{c}^{\top} \tilde{\mathbf{K}} \mathbf{c}
$$

The modal stiffness matrix $\tilde{\mathbf{K}}$ is found by computing the matrix multiplications and performing the integration. However, in order to substantially ease and reduce the number of computations, a reformulation is introduced. The fact that only the (diagonal) matrix $\boldsymbol{\Psi}$ depends on $z$ is utilised and therefore, only these matrices have to be integrated. We rearange the order of the terms in $\tilde{\mathbf{K}}$ to isolate the $\boldsymbol{\Psi}$-matrices within the integration. This is achieved by introducing the mathematical Hadamard product: $\circ$, which allows us to separate the matrices $\boldsymbol{\Psi}$ in the integration from the rest of the expression. Furthermore, to utilise the fact that $\boldsymbol{\Psi}$ contains values in its diagonal only, the Kronecker product: $\otimes$ is introduced. Hence, a combination of the Kronecker product and a re-formulation of $\boldsymbol{\Psi}$ into a column vector containing the diagonal components only, denoted $\hat{\mathbf{\Psi}}$, (see also [31]), the modal stiffness matrix is written as:

$$
\begin{gathered}
\tilde{\mathbf{K}}=\mathbf{T}_{c}^{\top}\left(\left[\begin{array}{c}
\mathbf{V} \\
\dot{\mathbf{V}}
\end{array}\right]^{\top}\left[\begin{array}{cc}
\mathbf{K}_{a a} & \mathbf{K}_{a b} \\
\mathbf{K}_{b a}^{\top} & \mathbf{K}_{b b}
\end{array}\right]\left[\begin{array}{c}
\mathbf{V} \\
\dot{\mathbf{V}}
\end{array}\right]\right. \\
\left.\circ \int_{0}^{\ell} \hat{\boldsymbol{\Psi}}^{\top} \otimes \hat{\mathbf{\Psi}} d z\right) \mathbf{T}_{c}
\end{gathered}
$$

With this formulation of $\tilde{\mathbf{K}}$, the integration is easily performed analytically due to the fairly simple amplitude functions by first computing the indefinite integral, and then using the fundamental theorem of calculus. 
As stated previously, the energy formulation in Equation (28) considers the vector of modal constants as variables or temporary modal degree of freedom vectors. In the next subsection, a transformation is introduced where the temporary modal degrees of freedom vectors are written in terms of nodal beam element degrees of freedom vectors at the boundaries, i.e. at each end of the beam.

\subsection{Beam element formulation}

This subsection formulates the beam element by considering the potential energy from Equation 25, the load contributions from Equation (26), and the strain energy written in modal form in Equation (28). At first, the strain energy shall be written in terms of beam element boundary degrees of freedom instead of the temporary modal degrees of freedom. Then, substituting into the potential energy, taking variation and by requiring the first variation of the energy to be stationary, we achieve the beam element formulation.

\subsubsection{Beam element degrees of freedom}

The beam element degrees of freedom are directly linked to the cross-sectional degrees of freedom at the boundaries. Therefore, considering the deformation vector $\mathbf{u}(z)$ and letting the position variable $z$ adopt the boundary values of the beam element, i.e. $z=0$ and $z=\ell$, where $\ell$ is the beam length, the deformations at the boundaries in the beam element degree of freedom vector $\mathbf{u}_{b}$ are expressed as:

$$
\mathbf{u}_{b}=\left[\begin{array}{l}
\mathbf{u}(0) \\
\mathbf{u}(\ell)
\end{array}\right]
$$

Substituting: $\mathbf{u}(z)=\mathbf{V} \boldsymbol{\Psi}(z) \mathbf{T}_{c} \mathbf{c}$, a part of Equation (24), we may write Equation 30 as:

$$
\mathbf{u}_{b}=\left[\begin{array}{c}
\mathbf{u}(0) \\
\mathbf{u}(\ell)
\end{array}\right]=\left[\begin{array}{ccc}
\mathbf{V} & \boldsymbol{\Psi}(0) & \mathbf{T}_{c} \\
\mathbf{V} & \boldsymbol{\Psi}(\ell) & \mathbf{T}_{c}
\end{array}\right] \mathbf{c}
$$

From this equation it can be seen that there is a relation between the deformations at the boundaries in $\mathbf{u}_{b}$ and the temporary modal degrees of freedom in c. This relation was in fact used in Equation (28). Consequently, a transformation matrix $\mathbf{A}$ is introduced and Equation (31) is re-written into:

$$
\mathbf{u}_{b}=\mathbf{A} \mathbf{c} \quad \text { where } \mathbf{A}=\left[\begin{array}{ccc}
\mathbf{V} & \boldsymbol{\Psi}(0) & \mathbf{T}_{c} \\
\mathbf{V} & \boldsymbol{\Psi}(\ell) & \mathbf{T}_{c}
\end{array}\right]
$$

With this formulation, $\mathbf{A}$ is defined as a squared, positive definite and invertible matrix that allows $\mathbf{c}$ to be written as:

$$
\mathbf{c}=\mathbf{A}^{-1} \mathbf{u}_{b}
$$

\subsubsection{Beam element stiffness matrix}

If Equation (33) is substituted into the modal strain energy formulation in Equation (28) we are able to express the beam element strain energy in terms of displacements at the boundaries. This is:

$$
\begin{aligned}
U & =\frac{1}{2} \mathbf{u}_{b}^{\top}\left[\mathbf{A}^{-1}\right]^{\top} \tilde{\mathbf{K}} \mathbf{A}^{-1} \mathbf{u}_{b} \\
& =\frac{1}{2} \mathbf{u}_{b}^{\top} \mathbf{K} \mathbf{u}_{b}
\end{aligned}
$$

in which the beam element stiffness matrix $\mathbf{K}$ is introduced as:

$$
\mathbf{K}=\left[\mathbf{A}^{-1}\right]^{\top} \tilde{\mathbf{K}} \mathbf{A}^{-1}
$$

\subsubsection{The beam element}

Substituting the strain energy from Equation 34 into the potential energy Equation (25) and the potential from the applied load in Equation (26) the full potential energy takes the form:

$$
V=U+\Pi=\frac{1}{2} \mathbf{u}_{b}^{\top} \mathbf{K} \mathbf{u}_{b}-\mathbf{u}_{b}^{\top} \mathbf{f}
$$

Then, taking the first variation in the displacement field, $\delta \mathbf{u}_{b}$, the potential energy becomes:

$$
\delta V=\delta \mathbf{u}_{b}^{\top} \mathbf{K} \mathbf{u}_{b}-\delta \mathbf{u}_{b}^{\top} \mathbf{f}
$$

and by requiring stationarity of the energy, it must equal zero for all variations $\left(\delta V=0\right.$ where $\delta \mathbf{u}_{b}^{\top} \neq \mathbf{0}$ ) whereby the well-known linear elastic beam formulation is derived:

$$
\mathbf{K} \mathbf{u}_{b}=\mathbf{f}
$$

With the formulation in Equation (37) a linear relation between the boundary loads $\mathbf{f}$, and the boundary deformations $\mathbf{u}_{b}$ is given - the beam element formulation.

The trivial solution procedure of the equation system in Equation (37) will not be expressed here, but reference is made to literature, such as Cook et al. 36]. In this literature, it is also described how aligned beam elements can be assembled. However, bear in mind that the presented formulation relies on exact longitudinal amplitude (shape) functions whereas traditional finite element formulations rely on approximations between end nodes through interpolation functions. Therefore, the presented beam element can adopt any length without further approximation.

\subsection{Boundary conditions}

The boundary conditions of a beam element are defined by the use of standard methods and operate on the deformation vector $\mathbf{u}_{b}$. In this deformation vector each degree of freedom within the two beam end cross-sections are represented. Therefore, it is possible to add boundary conditions related to each degree of freedom within an end cross-section. Furthermore, this allows the modelling of many kinds of supports, e.g. simple support conditions and clamped support conditions. However, support and load conditions should, if possible, be distributed to several nodes avoiding singularities and local stress concentration.

Advanced boundary conditions such as partially restrained cross-sections will not be described here, but special cross-section restraints can be added at the crosssection level, which however, is beyond the scope of this paper. 


\section{Element deformations, strains and stresses}

Having solved Equation (37), this section describes how to find displacements, deformation derivatives, strains, and stresses in an arbitrary point within a wall element of any cross-section within the thin-walled beam element.

\subsection{Deformation field}

The deformations at the beam boundaries are given by the beam element deformation vector $\mathbf{u}_{b}$ corresponding to the cross-sectional degrees of freedom at the beam element ends. Knowing these, the modal content can be determined using Equation (33). This enables us to determine the deformation vector and the axial derivative of the deformation vector of a cross-section at a given axial position $z$ using Equation (24). Thus, the cross-section deformation vector at the axial position $z$ can be found as:

$$
\left[\begin{array}{c}
\mathbf{u}(z) \\
\dot{\mathbf{u}}(z)
\end{array}\right]=\left[\begin{array}{c}
\mathbf{V} \\
\dot{\mathbf{V}}
\end{array}\right] \boldsymbol{\Psi}(z) \mathbf{T}_{c} \mathbf{A}^{-1} \mathbf{u}_{b}
$$

From these cross-section deformation vectors the local wall element degrees of freedom $\mathbf{u}_{w}^{e l}$ and $\mathbf{u}_{\Omega}^{e l}$ as well as the corresponding axial derivatives $\dot{\mathbf{u}}_{w}^{e l}$ and $\dot{\mathbf{u}}_{\Omega}^{e l}$ can be extracted.

The local deformations in any point $(n, s)$ of a wall element in the cross-section at the axial position $z$ may now be found using Equation (4).

\subsection{Strain field}

Using the element wall deformations and axial deformation derivatives of a cross-section at the axial position $z$, the local strains at any point of a wall element is found as follows:

$$
\begin{aligned}
\varepsilon_{s s}^{e l}(n, s, z)= & {\left[\mathbf{N}_{s, s}-n \mathbf{N}_{n, s s}\right] \mathbf{u}_{w}^{e l} } \\
\varepsilon_{z z}^{e l}(n, s, z)= & {\left[\mathbf{N}_{\Omega}+n \mathbf{N}_{\alpha}\right] \dot{\mathbf{u}}_{\Omega}^{e l} } \\
\gamma_{n z}^{e l}(s, z)= & \mathbf{N}_{n} \dot{\mathbf{u}}_{w}^{e l}+\mathbf{N}_{\alpha} \mathbf{u}_{\Omega}^{e l} \\
\gamma_{s z}^{e l}(n, s, z)= & {\left[\mathbf{N}_{s}-n \mathbf{N}_{n, s}\right] \dot{\mathbf{u}}_{w}^{e l} } \\
& +\left[\mathbf{N}_{\Omega, s}+n \mathbf{N}_{\alpha, s}\right] \mathbf{u}_{\Omega}^{e l}
\end{aligned}
$$

in which only the element wall deformation vectors $\mathbf{u}_{w}^{e l}$ and $\mathbf{u}_{\Omega}^{e l}$ and their axial derivative $\dot{\mathbf{u}}_{w}^{e l}$ and $\dot{\mathbf{u}}_{\Omega}^{\text {el }}$ are dependent on the axial position coordinate $z$.

\subsection{Stress field}

Knowing the cross-sectional strain field for a given $z$ value, the corresponding stresses can be computed using the linear elastic constitutive relations for isotropic materials presented in Equation (7). Substituting the strains into the constitutive relations gives us the stresses in each wall element as a function of the local coordinates as follows:

$$
\begin{aligned}
\sigma_{s s}^{e l}(n, s, z)=E_{s} & \left(\left[\mathbf{N}_{s, s}-n \mathbf{N}_{n, s s}\right] \mathbf{u}_{w}^{e l}\right. \\
& \left.+\nu\left[\mathbf{N}_{\Omega}+n \mathbf{N}_{\alpha}\right] \dot{\mathbf{u}}_{\Omega}^{e l}\right) \\
\sigma_{z z}^{e l}(n, s, z)=E_{s} & \left(\left[\mathbf{N}_{\Omega}+n \mathbf{N}_{\alpha}\right] \dot{\mathbf{u}}_{\Omega}^{e l}\right. \\
& \left.+\nu\left[\mathbf{N}_{s, s}-n \mathbf{N}_{n, s s}\right] \mathbf{u}_{w}^{e l}\right) \\
\tau_{n z}^{e l}(s, z)=G( & \left.\mathbf{N}_{n} \dot{\mathbf{u}}_{w}^{e l}+\mathbf{N}_{\alpha} \mathbf{u}_{\Omega}^{e l}\right) \\
\tau_{s z}^{e l}(n, s, z)=G( & {\left[\mathbf{N}_{s}-n \mathbf{N}_{n, s}\right] \dot{\mathbf{u}}_{w}^{e l} } \\
& \left.+\left[\mathbf{N}_{\Omega, s}+n \mathbf{N}_{\alpha, s}\right] \mathbf{u}_{\Omega}^{e l}\right)
\end{aligned}
$$

Distinguishing between membrane and bending stresses is common. However, this will not be incorporated directly, but could easily be achieved from Equation 40 by separating into terms that are dependent or independent of the $n$-coordinate.

\subsection{Exponential decay}

A characteristic of those modes having an exponential amplitude function is that they are either derived as real couples or complex quadruples. A real couple represents a displacement mode with an increasing amplitude along the beam axis peaking at the one end, and a mode with a decaying amplitude peaking at the other end. In case of a complex set of eigenvalues, there will be a real and an imaginary cross-section mode vector with an increasing or decreasing harmonic oscillation. This corresponds to two mode shapes with a decaying amplitude function and two modes with an increasing amplitude function, respectively.

Due to this characteristic feature related to the exponential modes and their axial decay behaviour, it becomes interesting to assess their attenuation length. The real part of the eigenvalue determines the attenuation length. The higher the eigenvalue, the shorter the attenuation length is. Hence, an attenuation length can be defined as:

$$
L_{a}=\frac{\epsilon}{|\operatorname{Re}(\lambda)|}
$$

in which $\epsilon$ defines a lower level of interest where the decay has lead to a diminished displacement, which could be taken as $5 \%$ of the peak value. This results in $\epsilon$ being around three. Jönsson [39] introduces a value of $\epsilon=\pi$ giving a lower level of: $e^{-\pi} \approx 4 \%$. Giavotto et al. [40] use a value of $\epsilon=3$, which results in: $e^{-3}=5 \%$ as the lower level. The use of $\epsilon=\pi$ is relevant due to the equivalence to half the harmonic wavelength or a buckling length.

Depending on the attenuation length, the displacement modes found in Equation 21 can be characterised as global distortional modes or local distortional plate modes. The latter has an attenuation length typically less than the main cross-section dimension, whereas the former often has an attenuation length being several times the crosssection dimension. 


\section{Examples}

In this section, four examples are used to illustrate and assess the results of the presented thin-walled beam element formulation. Both the geometry and the type of load vary in the four examples:

1. A cantilever having a hollow rectangular box section loaded in shear. This example illustrates the deformation behaviour, the normal stresses and shear stresses, as well as the shear stress transition at cross-section corners.

2. An open channel section with a non-symmetric transverse load, which induces torsional deformations, torsional warping stresses and bending stresses.

3. A short simply supported box section loaded in bending and exhibiting shear lag with non-linear normal stresses in the flanges.

4. The final example is a longitudinal assembly of three similar channel elements with a distortional load. The attenuation length related to exponential modes, mode intensities, and transverse bending stresses are assessed.

For each example, both stresses and nodal displacements will be compared to results obtained with the commercial finite element program Abaqus [41. The finite element analysis is performed on a model with isotropic material and four node shell elements (S4-shell element in Abaqus syntax) using full integration. Furthermore, the linear elastic finite element calculations are based on a structured rectangular mesh with a side length of $5 \mathrm{~mm}$.

\subsection{Ex. 1 - Tip loaded cantilever box section}

The first example considers a cantilevered beam with a closed rectangular cross-section as shown in Figure 5, in which the parameters of the example also are listed. The load at the tip is applied as evenly distributed line loads along the two webs of the end cross-section in Abaqus and as nodal loads in the theory presented here. Solving the beam element formulation from Equation (37) and then determining the deformation using Equation (38) renders a deformation as illustrated to the left in Figure 6 mainly having flexural bending deformation. In Table 2 nodal displacements are listed, which are extracted from the present theory and from the analysis using the commercial finite element program. Furthermore, a comparison between the nodal displacements and their relative deviation is given in Table 2. The values are extracted from the nodes at the free end $(Z=\ell)$ in the upper and lower right corners of the cross-section. In the vertical direction, a relative derivation of $0.10 \%$ is seen. If the horizontal displacements in $u_{x}$ are compared, given in the table, a relatively high deviation is seen but at the same time the displacements are small, and therefore this deviation corresponds to $0.1 \cdot 10^{-3} \mathrm{~mm}$ which is equal to three millions of the
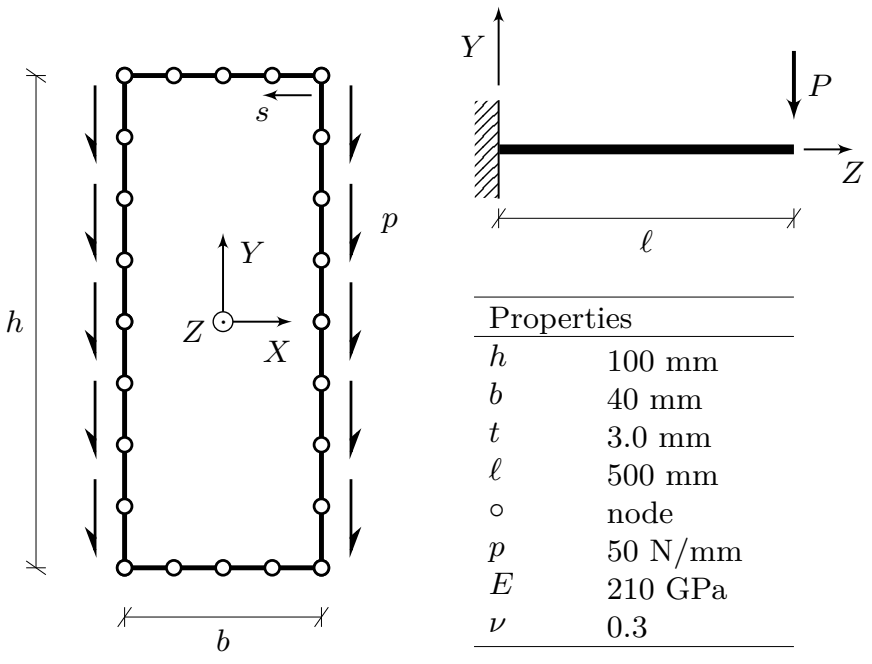

\begin{tabular}{ll}
\hline \multicolumn{2}{l}{ Properties } \\
\hline$h$ & $100 \mathrm{~mm}$ \\
$b$ & $40 \mathrm{~mm}$ \\
$t$ & $3.0 \mathrm{~mm}$ \\
$\ell$ & $500 \mathrm{~mm}$ \\
$\circ$ & node \\
$p$ & $50 \mathrm{~N} / \mathrm{mm}$ \\
$E$ & $210 \mathrm{GPa}$ \\
$\nu$ & 0.3 \\
\hline
\end{tabular}

Figure 5: Ex. 1 - discretisation and geometrical properties of the cantilever beam with a closed box section including local set-up and general load/support configurations

width only. The $u_{x}$-deformations represent a contraction of the top flange and a widening of the lower flange. This is a result of the Poisson effect since the top flange is exposed to axial tension and the bottom flange is in axial compression.

The stresses will be analysed next and for this purpose, the cross-section at mid-span $(Z=\ell / 2)$ is assessed in order to alleviate the local effects that may occur near loaded or supported nodes. To the right in Figure 6, a threedimensional illustration of the normal stresses is shown and in Figure 7] the values found at mid-span are seen. The axial stresses are obtained along the centre line of the cross-sectional wall elements. A good agreement is seen between the two models with a maximum relative deviation of $-0.54 \%$ found at the corners.

To be assessed next are the shear stresses. Due to the kinematics and the constitutive relations in Equation (7), we have $\tau_{s z}$-stresses as well as $\tau_{n z}$-stresses. On the other hand, the shell model in Abaqus considers $\tau_{s z}$-stresses only. Comparing the $\tau_{s z}$-stresses yields overall, good similarities and the relative deviation of the largest $\tau_{s z}$-stresses is
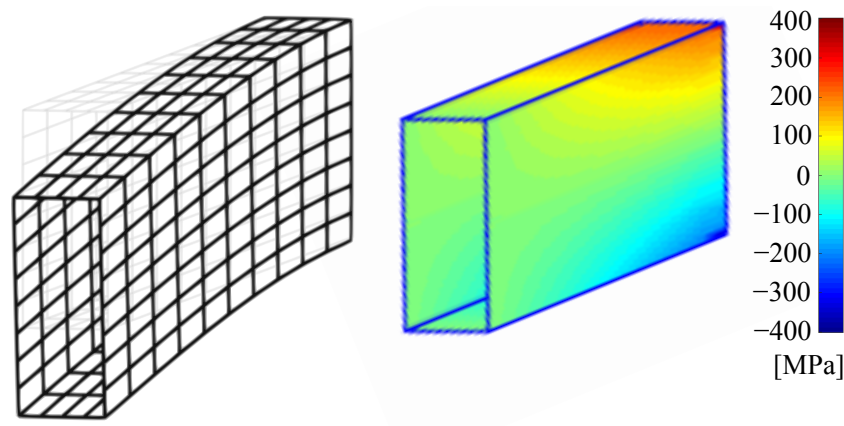

Figure 6: Ex. 1 - Deformation and $\sigma_{z z}$-stresses. Deformations are scale by a factor 20 
Table 2: Ex. 1 - Nodal deformations at the upper and lower right corners comparing the two models. Measurements in $[\mathrm{mm}]$

\begin{tabular}{c|ccc}
\hline $\begin{array}{c}\left(\frac{b}{2}, \pm \frac{h}{2}, \ell\right) \\
(n=0)\end{array}$ & $\begin{array}{c}\text { Present } \\
\text { theory }\end{array}$ & Abaqus & $\begin{array}{c}\text { Relative } \\
\text { deviation }\end{array}$ \\
\hline$u_{x}$ & \pm 0.0013 & \pm 0.0012 & $-8.33 \%$ \\
$u_{y}$ & -1.9060 & -1.9080 & $0.10 \%$ \\
$u_{z}$ & \pm 0.2711 & \pm 0.2712 & $-0.04 \%$ \\
\hline
\end{tabular}

around $0.15 \%$ depending on the mesh density. Figure 8 illustrates the shear stresses at $Z=\ell / 2$ and for $n=0 \mathrm{~mm}$, i.e. the middle surface of the wall. The different mesh densities illustrated in Figure 8 have been considered to investigate the local shear effects near the corners. Thus, in the upper flange, two, four, six and eight elements are used as shown from the left to the right in the figure. Especially the $\tau_{n z}$-plots indicate that the stresses are locally peaking near the corners. Additionally, if comparing the $\tau_{s z}$-stresses obtained to those from Abaqus, deviations are significant at corners. However, with an increased mesh density this effect becomes more isolated near the corners. This local effect has inspired us to perform a detailed investigation of the stress transmission around corners. Therefore, a solid finite element model is created in Abaqus as well. In this model, an eight-node linear brick element (C3D8 in Abaqus syntax) has been used to discretise the beam in a structured mesh. A section cut-out of the upper right corner at mid-span is illustrated in Figure 9 and the stress variations along the middle of the walls are likewise shown here. From Figure 9 it can be seen how the stress variations follows the same pattern as the one obtained considering the present theory (Figure 8). However, Figure 9 also indicates that the decay of the $\tau_{n z}$-stresses is less than two times the wall thickness. Therefore, the results from this solid finite element model indicate exactly what
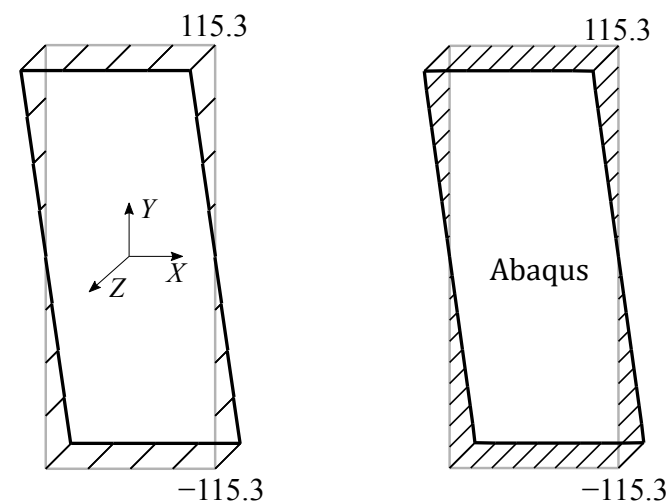

Figure 7: Ex. 1 - Axial stresses, $\sigma_{z z}$, in $[\mathrm{MPa}]$ at $Z=\ell / 2$ and $n=0 \mathrm{~mm}$. To the left stresses found from the theory presented here, to the right stresses extracted from Abaqus was seen in the stress distributions in Figure 8 This result supports that small elements near the corners should be considered when adapting the present theory in combination with this type of wall element. An example of this is illustrated in Figure 10 showing the results of an analysis with an element mesh having small wall elements at the corners only. 


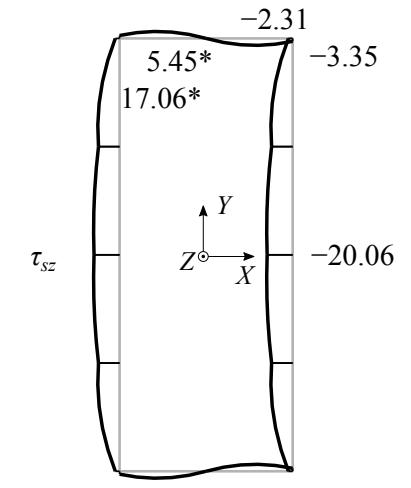

Wall elements in flange:

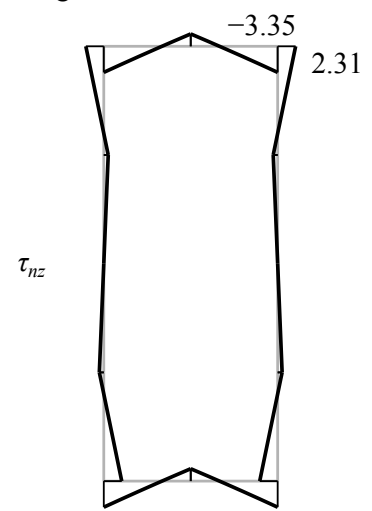

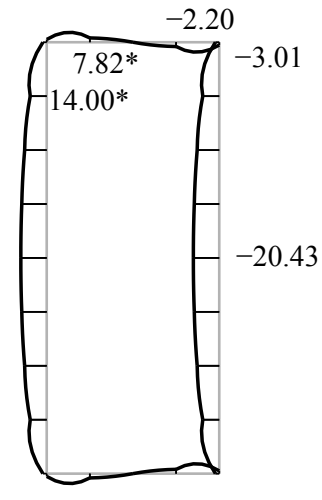

4

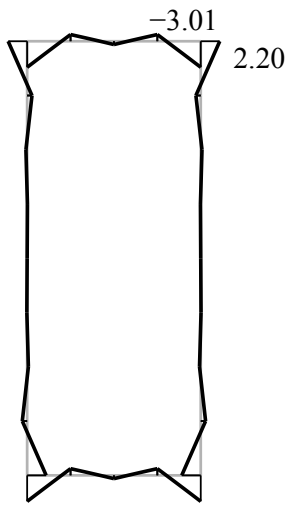

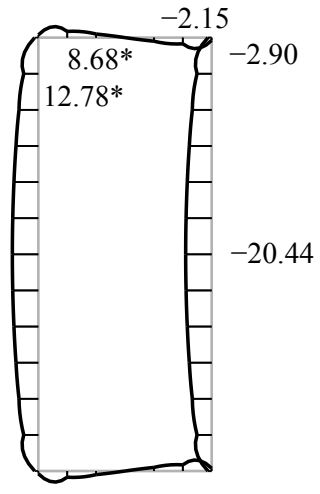

6

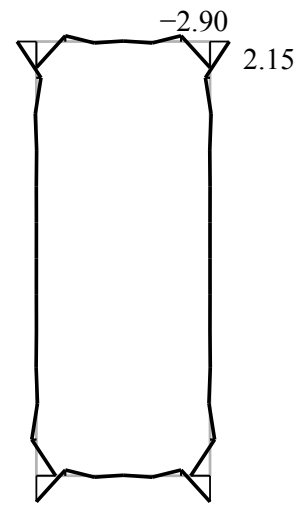

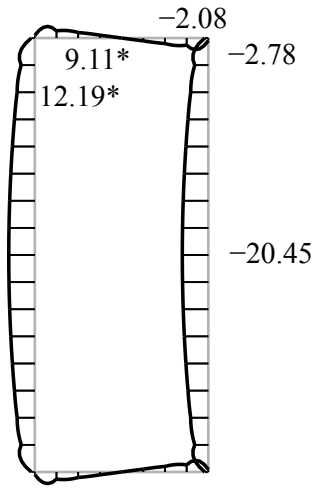

8

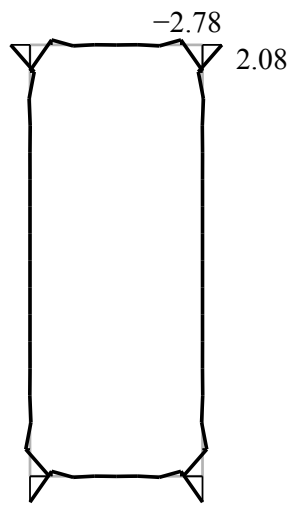

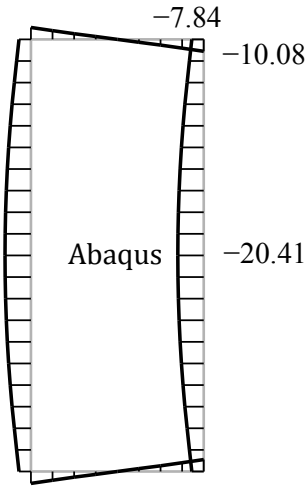

8

Figure 8: Ex. 1 - Shear stresses at $n=0 \mathrm{~mm}$ and $Z=\ell / 2$ with an increasing mesh density. Also including stresses subtracted from the finite element analysis in Abaqus. Stresses given in [MPa]. The $\tau_{s z}$-stresses marked with an asterisk indicates the highest stress value obtained in the wall element closest to the corner either in the web or flange (the stress is computed at teen points along each wall element) 


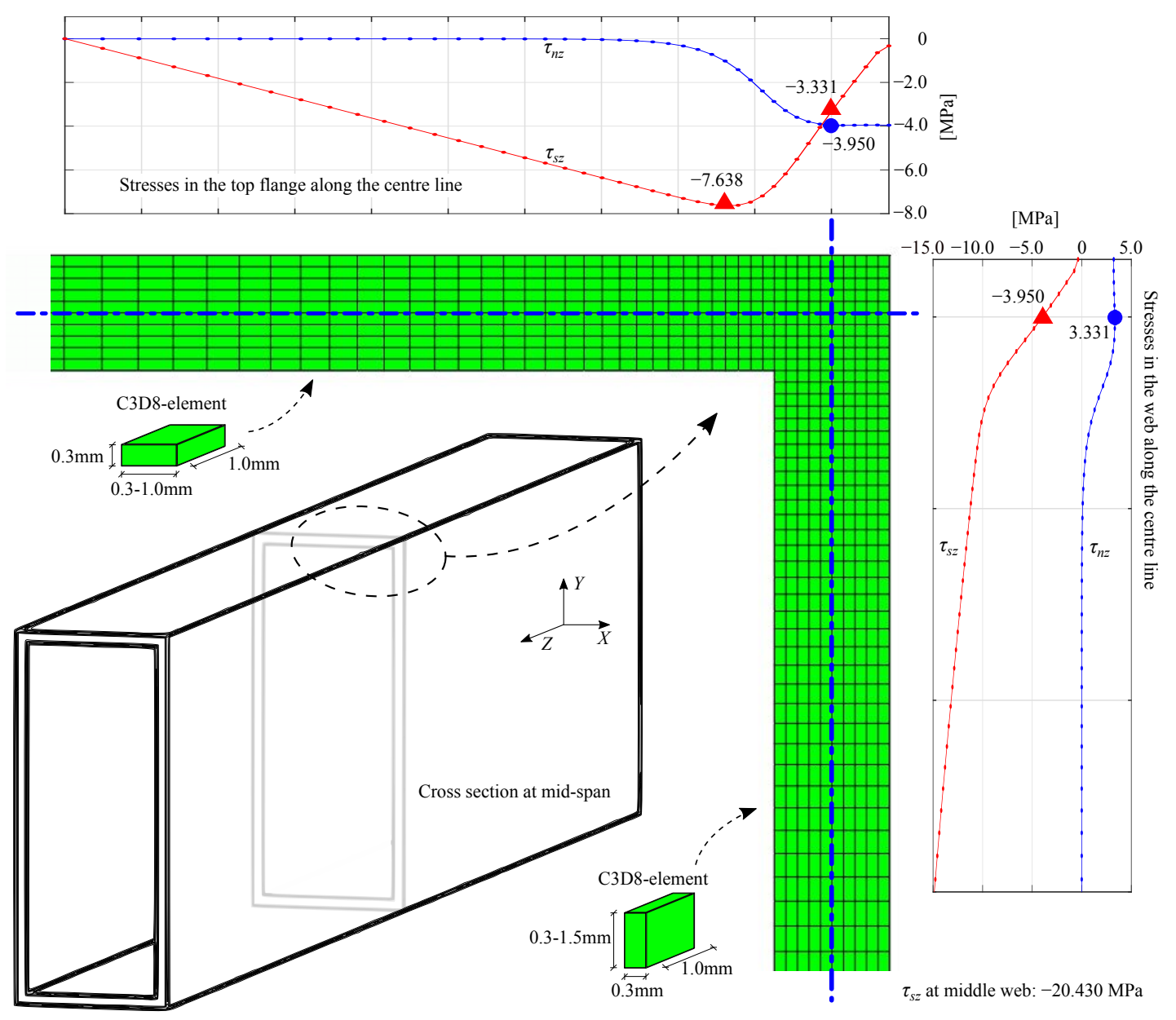

Figure 9: Ex. 1 - Results from the Abaqus model using solid elements 

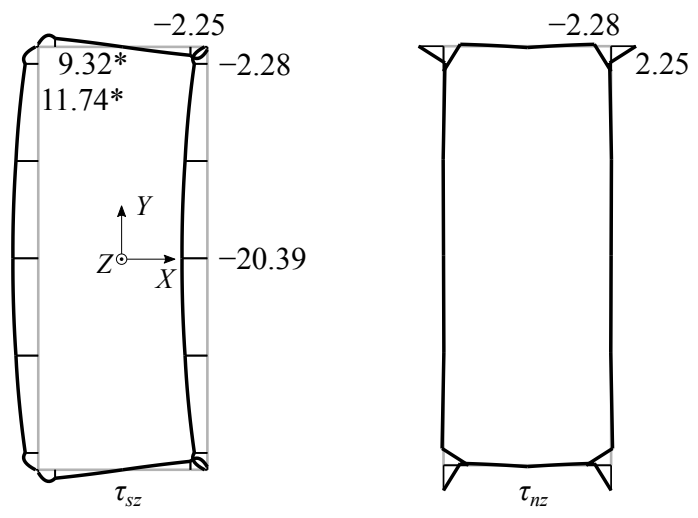

Figure 10: Ex. 1 - Shear stresses using a special mesh with small elements near corners. Stresses in $[\mathrm{MPa}]$ at $Z=\ell / 2$ and $n=0 \mathrm{~mm}$

\subsection{Ex. 2-Shear loaded cantilevered channel section}

This example examines a mono-symmetric cantilever channel section exposed to a load which induces flexural and torsional displacements. The load configurations, as well as the geometrical properties, are shown in Figure 11 In the present implementation, the end cross-section load is applied as point loads distributed to each node in the web and in the finite element program Abaqus as a line load acting on the web. The clamped boundary condition is modelled by restraining all degrees of freedom within the end cross-section. Due to the load configuration, the beam element deforms partly in bending and partly in torsion as illustrated in Figure 12

This example is chosen such that it includes the added effects of classic flexure, torsion and torsional warping. The channel section is loaded along the web and thus, the beam is expected to flexure about the strong principle axis and twists about the shear centre. Furthermore, since the beam is cantilevered with a completely clamped bound-
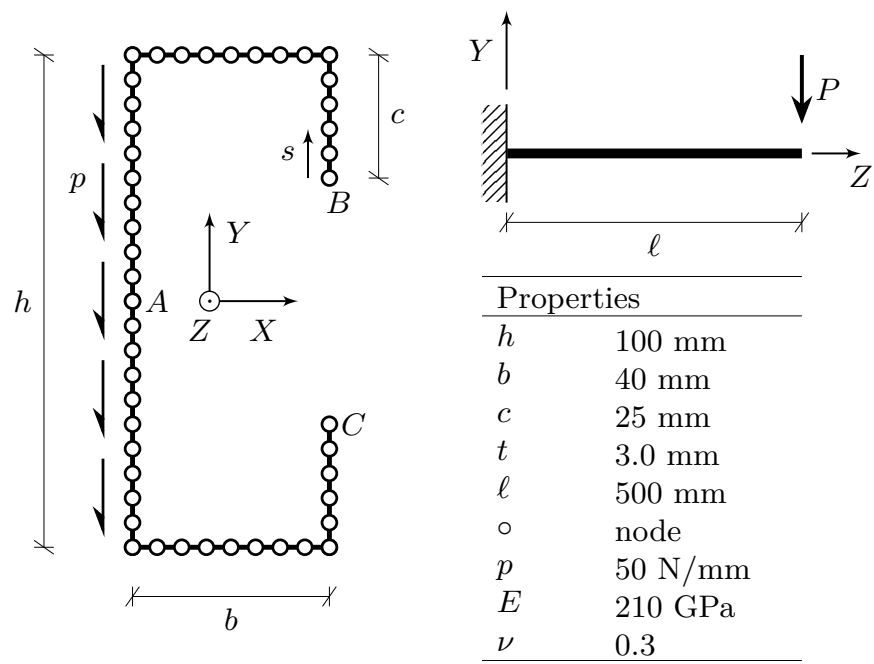

Figure 11: Ex. 2 - Geometric properties, discretisation, and load properties of the shear loaded channel section

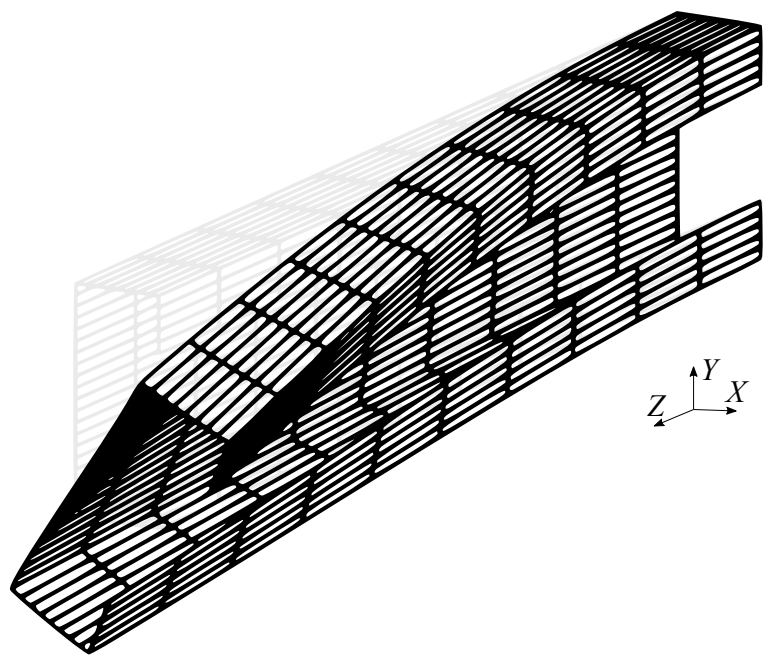

Figure 12: Ex. 2 - Three-dimensional deformation of the cantilever drawn with a scaling factor equal 20

ary condition at one end, the beam will exhibit torsional warping. Therefore, it is chosen to compare the resulting displacements at the free end and the stresses close to the clamped end of the beam with those found using finite shell elements.

Let us first compare the displacement of the beam end at the central node point $A$ in the web to an Abaqus shell model. The points $A, B$ and $C$ are shown in Figure 11 First of all, the displacements in $u_{x}$ and $u_{z}$ of point $A$ vanish in both models. The present theory results in a vertical displacement of $u_{y}=-1.8392 \mathrm{~mm}$ and Abaqus results in $u_{y}=-1.8473 \mathrm{~mm}$ giving a relative deviation of the present theory compared to Abaqus of $0.44 \%$. The influence of torsion and the related warping can be illustrated by simply comparing the nodal displacements of the outer most free edge point of the lip, i.e. at point $B$. In Table 3 , the three displacements of the upper lip are given. It can be seen that the maximum relative deviation of the displacements at this nodal point $B$ is $0.87 \%$ and it is related to the axial warping displacements of warping and flexure. Similar displacements are of course found at point $C$ in the lower lip, however, $u_{x}$ and $u_{z}$ change signs.

Next, the normal stress and shear stresses of the model are compared to those found using an Abaqus shell model.

Table 3: Ex. 2 - The nodal displacements in $[\mathrm{mm}]$ of the free end of the upper lip at point $B$, i.e. at $Z=500 \mathrm{~mm}$.

\begin{tabular}{r|rrc}
\hline & $\begin{array}{r}\text { Present } \\
\text { theory }\end{array}$ & Abaqus & $\begin{array}{r}\text { Relative } \\
\text { deviation }\end{array}$ \\
\hline$u_{x}$ & 1.1055 & 1.1107 & $0.47 \%$ \\
$u_{y}$ & -2.9155 & -2.9324 & $0.58 \%$ \\
$u_{z}$ & -0.1488 & -0.1501 & $0.87 \%$ \\
\hline
\end{tabular}



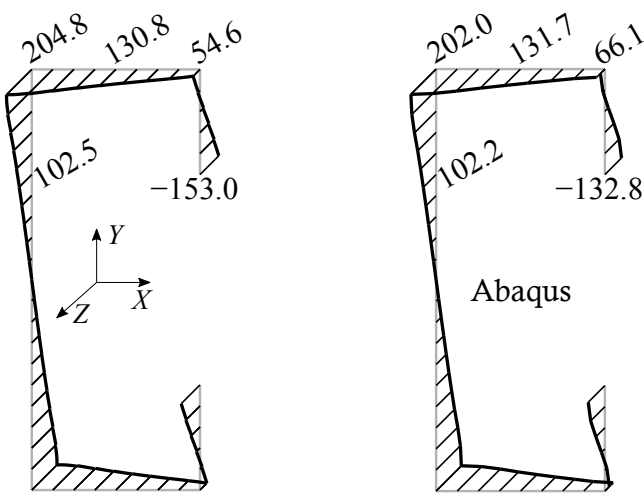

Figure 13: Ex. 2 - A comparison of normal stresses, $\sigma_{z z}$ in [MPa], measured $10 \mathrm{~mm}$ from the fixed end, i.e. at $Z=10 \mathrm{~mm}$, and $n=0 \mathrm{~mm}$
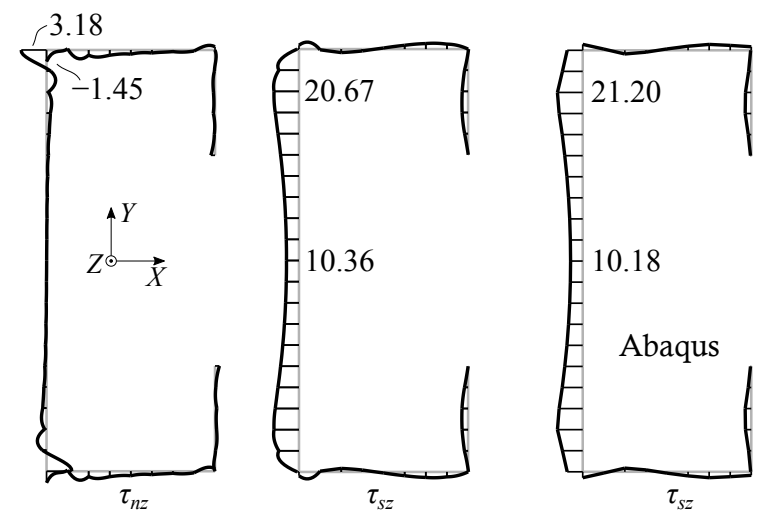

Figure 14: Ex. 2 - The shear stresses are shown in [MPa] at a cross-section $10 \mathrm{~mm}$ from the support, i.e. $Z=10 \mathrm{~mm}$, moreover, $n=0 \mathrm{~mm}$

Since the modelling of displacements and therefore also shear stresses are quite different at section corners and therefore difficult to compare, especially at the fixed end, we have chosen to compare stresses that occur quite close to the fixed end, having $Z=10 \mathrm{~mm}$. The mid surface normal stresses measured $10 \mathrm{~mm}$ from the fixed end are shown in Figure 13 and the stress values are given at the crosssection corners, at the lip end, at the web quarter point, and at the centre of the flange. This clearly shows that there are torsional warping stresses and bending stresses of the beam. Comparing the stresses obtained using Equation (40) and those from Abaqus it is seen that there is some difference, which is maximised at the upper right corner with a relative deviation of $-17.4 \%$.

The shear stresses that occur $10 \mathrm{~mm}$ from the fixed end are shown in Figure 14 In general, the $\tau_{s z}$-stresses have a similar distribution in both models.

Overall, the results obtained using the two different models agree. This despite the fact that the theory presented includes 564 degrees of freedom only, whereas the shell finite element model in Abaqus includes 28482 degrees of freedom. Indeed, this is due to the use of exact axial beam interpolation functions since the discretisation of the cross-section in the two models coincide.

\subsection{Ex. 3 - Box section exposed to shear lag}

This example assesses the effect of shear lag, which, for example, can be seen in relatively short thin-walled members with relative wide flanges. Consequently, this example examines a simply supported closed box beam with wide flanges.

The effect of shear lag is typically seen in box bridge sections with wide flanges and short spans. The shear lag effect is often of importance near supports (for example for uniformly loaded multi span members) and also near other large local transverse loads. This is because the shear with the associated shear deformation (warping) has to "transfer" the effects of the shear force (local transverse load) out into the flanges in order to build up the normal stresses needed for equilibrium. This effect is not included in classic theories of torsional beam theories, but it is included in the present model.

In this example, the beam is exposed to bending about the weak axis by a central transverse load. The load is applied on the central cross-section as line loads acting along the webs as shown in Figure 15 . The figure also illustrates the cross-section and gives the necessary parametric values.

The boundary conditions are introduced as a restriction of the in-plane translations at the two end cross-sections, and as an axial restraint to a single node within the crosssection at $Z=0 \mathrm{~mm}$. The rotational degrees of freedom are not restrained, and the load is distributed and applied as concentrated nodal loads in both models.

The vertical displacement of the lower left corner node at mid-span of the present model is compared to the results of an Abaqus shell model. Using the presented model a
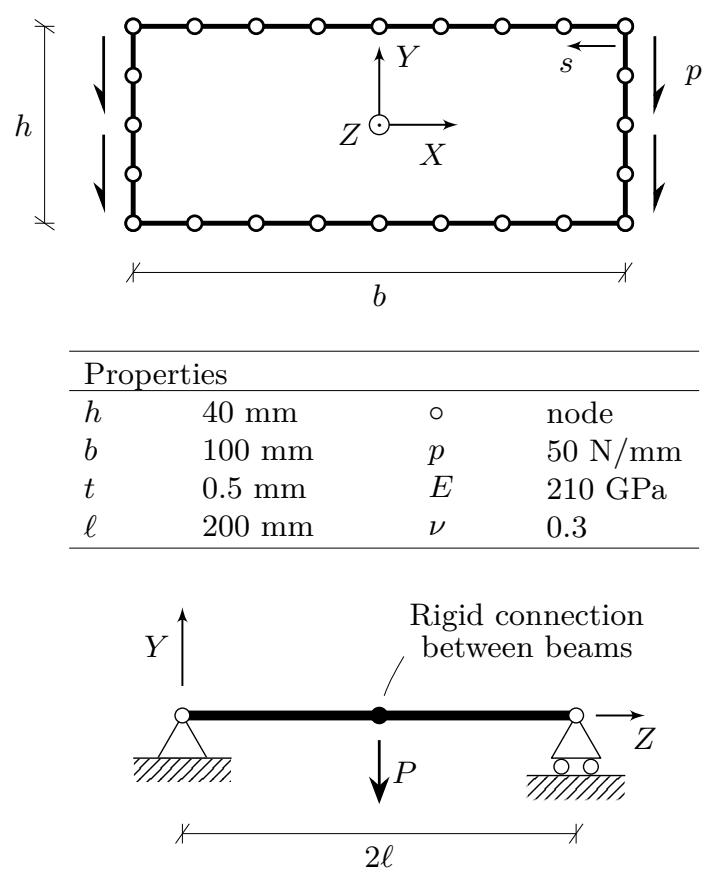

Figure 15: Ex. 3-General configurations used in this example 

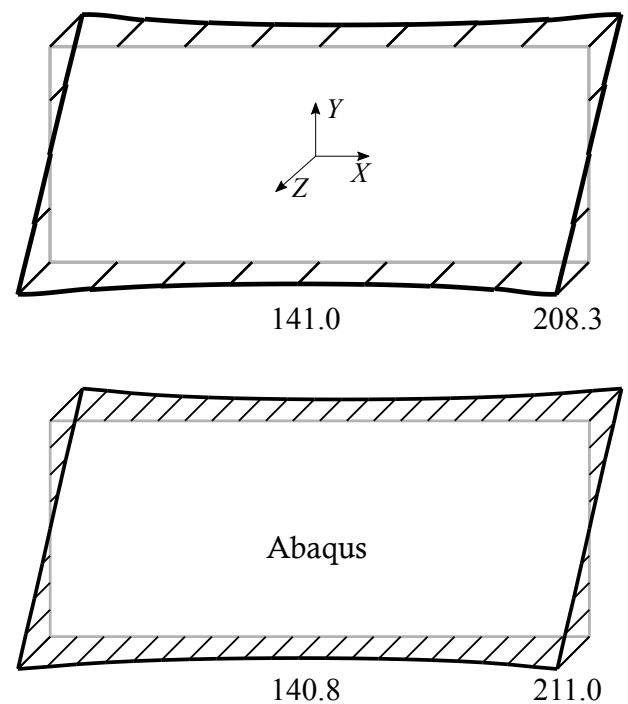

Figure 16: Ex. 3 - Normal stresses in [MPa] measured $10 \mathrm{~mm}$ from the middle of the beam, i.e. at $Z=190 \mathrm{~mm}$

vertical displacement of $u_{y}=0.7325 \mathrm{~mm}$ is found and using Abaqus a vertical displacement of $u_{y}=0.7408 \mathrm{~mm}$ is found with a relative deviation of $1.12 \%$.

The normal stress variation in a cross-section situated $10 \mathrm{~mm}$ from the centre of the beam is used to assess whether the present theory will cover shear lag. Hence, the stress variations in Figure 16 shows that the present theory covers shear lag. A relative reduction in the normal stresses between the flange middle and the corner is $32.3 \%$. If the results are compared to those obtained from the commercial finite element software Abaqus the maximum relative deviation is found to be $1.28 \%$ at the corners.

\subsection{Ex. 4-Open lipped channel section}

This final example considers a simply supported beam with an open channel section. The beam is assembled by three similar beam elements each of length $\ell$. The beam is loaded at: $Z=2 \ell$ with a point load at the end of each lip. The cross-section parameters, cross-section discretisation, and load set-up are shown in Figure 17. The support conditions are achieved by prohibiting any transverse deformations at each end. Furthermore, at the one end, a single node is restricted to have no axial deformation. The rotational degrees of freedom at the two ends are kept as free variables.

The overall deformation is illustrated in Figure 18 It corresponds to a global bending behaviour combined with a local deformation close to the loaded nodes. A cut-out side-view near the loaded nodes is illustrated in Figure 19. This figure shows clearly how a global bending and local deformations influence the deformed shape. Besides, the figure highlights how local deformations decay over a certain distance.

Let us in the following look into the behaviour of the central beam element. First of all, the constants computed by Equation (33) are found. They determine how the crosssectional displacement modes are linearly combined based on the intensities given by the components of $\mathbf{c}$. The constants are illustrated in Figure 20 where the absolute real values are given above the horizontal axis, and the absolute imaginary parts are given below the horizontal axis,

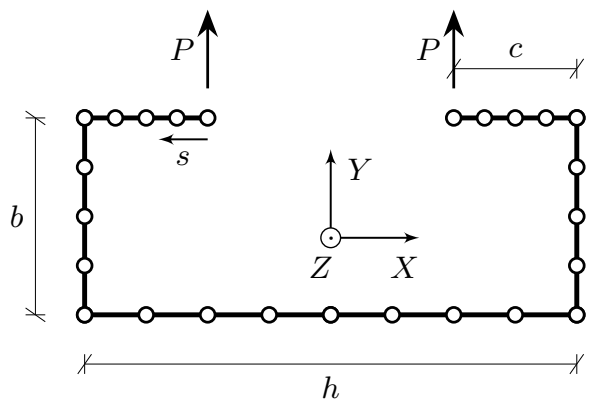

\begin{tabular}{llll}
\hline \multicolumn{3}{l}{ Properties } \\
\hline$h$ & $100 \mathrm{~mm}$ & $\circ$ & node \\
$b$ & $40 \mathrm{~mm}$ & $P$ & $1 \mathrm{kN}$ \\
$c$ & $25 \mathrm{~mm}$ & $E$ & $210 \mathrm{GPa}$ \\
$t$ & $3.0 \mathrm{~mm}$ & $\nu$ & 0.3 \\
$\ell$ & $500 \mathrm{~mm}$ & & \\
\hline
\end{tabular}

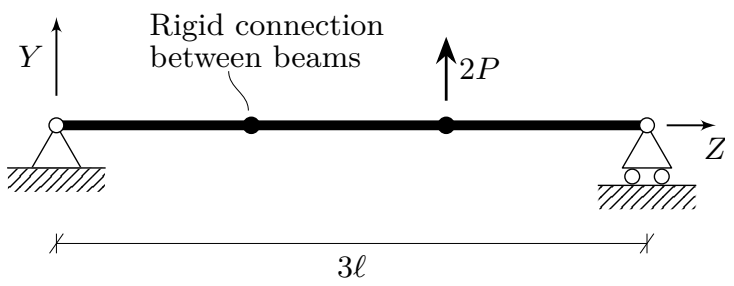

Figure 17: Ex. 4 - Open profile with simple supports, properties and overall static system 


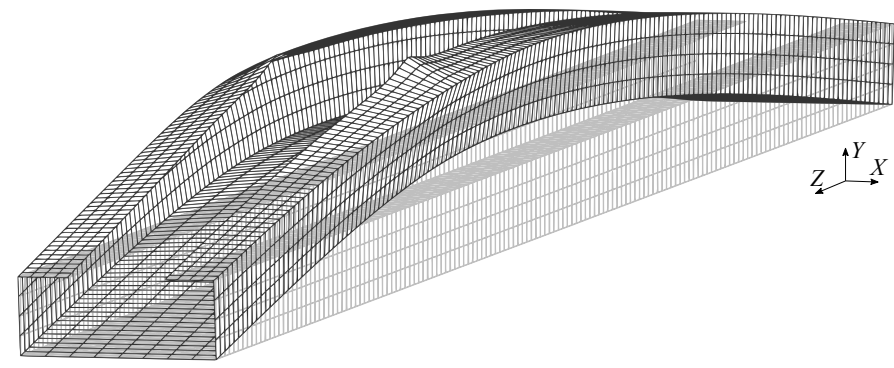

Figure 18: Ex. 4 - Three-dimensional visualisation of the deformation corresponding to the simply supported beam with two single loads attached to its lips. The deformation is scaled by a factor of 20

$80 \mathrm{~mm}$

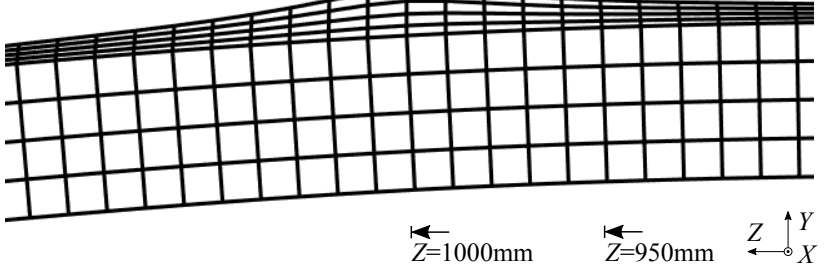

Figure 19: Ex. 4 - Decay length seen in the $Y, Z$-plane

respectively. The first twelve constants correspond to the fundamental modes and should always be considered. In Table 4 the most pronounced constants are listed with respect to the exponential modes.

To assess those modes contributing to the deflected shape, focus will be on the cross-section where the loads are applied $(Z=1000 \mathrm{~mm})$. In addition, only the transverse displacement part of the modes will be considered. The total transverse deformation of the cross-section is given in Figure 21a, which is found computing: $\mathbf{u}(z)=$ $\mathbf{V} \Psi(z) \mathbf{T}_{c} \mathbf{c}$. Figure 21b and 21; illustrates the contributions from the polynomial and exponential modes, respectively. Hence, the polynomial modes contribute with a rigid vertical deformation whereas the exponential modes gives the distortional deformations.

From the graph in Figure 20, it was indicated that not all modes were activated and Table 4 listed the eight most pronounced exponential modes. If these eight modes are

Table 4: Ex. 4 - The eight highest constants with respect to the exponential modes regarding beam segment two (written in a decreasing order)

\begin{tabular}{ccc}
\hline mode $i$ & $c_{i}$ & $L_{a}[\mathrm{~mm}]$ \\
\hline 23 & 0.655 & 84.28 \\
15 & $0.214+0.267 i$ & 540.90 \\
16 & $0.214-0.267 i$ & 540.90 \\
45 & $-0.173+0.109 i$ & 38.13 \\
46 & $-0.173-0.109 i$ & 38.13 \\
31 & $0.141-0.057 i$ & 79.75 \\
32 & $0.141+0.057 i$ & 79.75 \\
53 & 0.123 & 31.52 \\
\hline
\end{tabular}

added together the shape shown in Figure $21 \mathrm{~d}$ is achieved and it is very close to the one including all 288 exponential modes in Figure 21k. Now, a further assessment of these eight exponential modes are illustrated in Figure 22a to 22 . . In these figures the light grey deformed cross-section represents the combined shape from Figure 21d. The deformation in Figure $22 f$ is a combination of all remaining exponential modes showing a very small contribution. This confirms that the primary exponential deformation is a result of the eight selected modes. Figure 22b, 22 $\mathrm{k}$ and $22 \mathrm{~d}$ are contributions from three pairs of complex modes, which becomes real when they are added together in pairs.

For each of the exponential modes, an axial attenuation length may be found considering Equation (41) letting $\epsilon=\pi$. These attenuation lengths are listed in Table 4 Hence, mode 23 has an attenuation length: $L_{a}=84.28 \mathrm{~mm}$ that corresponds very well with the observed deformation pattern seen in Figure 19. The modes 15 and 16 are those having the largest decay length, which harmonise well with the cross-sectional deformation seen in Figure $22 \mathrm{~b}$.

Next, let us perform a comparison between the obtained stresses using Equation (40) and those obtained from the shell model in Abaqus. To avoid effects from singularities, a cross-section located $50 \mathrm{~mm}$ from the loaded nodes is analysed, which is at $Z=950 \mathrm{~mm}$. The axial stresses along the cross-sectional wall centre lines are shown in Figure 23 A maximum relative deviation of $0.30 \%$ is found at the upper right corner whereas the relative deviation at the web middle is only $-0.08 \%$. Due to the load configurations, transverse bending stresses will occur. Figure 24 illustrates the distribution of these obtained at the outer surface. Since the transverse stresses are interpolated linearly within each element, the found stress distribution is discontinuous between elements. The stresses obtained from Abaqus are extrapolated averaged nodal stresses, which therefore are continuous. Nonetheless, the overall shapes are similar, and the stresses deduced from the two models coincide with a relative deviation between $-0.37 \%$ and $-4.66 \%$.

\section{Discussion}

The formulation of an advanced thin-walled beam element has been presented. The beam element facilitates not only classic displacement patterns with rigid cross-section movements but also in-plane displacements related to the Poisson effect as well as distortional deformations with associated warping modes.

One of the main advantages obtained with this thinwalled beam theory is the use of exact beam interpolation functions in the axial direction. This allows the beam element to be used for any lengths. Consequently, a beam element is not limited to a finite length, which is the case in GBT since polynomial shape functions govern the axial variation.

Thus, the presented beam theory only has nodes and degrees of freedom at its end cross-sections and therefore, 


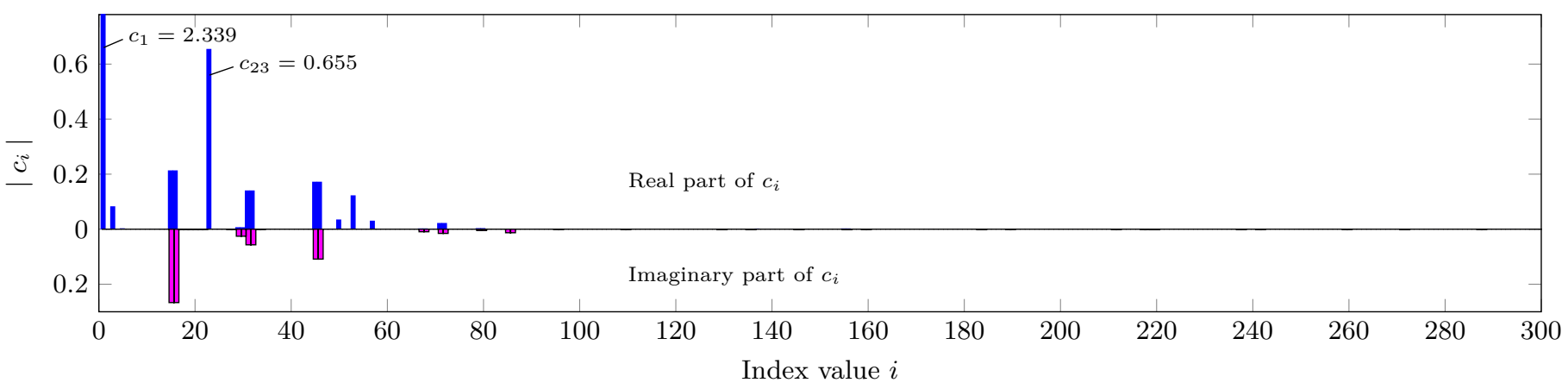

Figure 20: Ex. 4 - The absolute values of the real and imaginary part of the constants in c, respectively

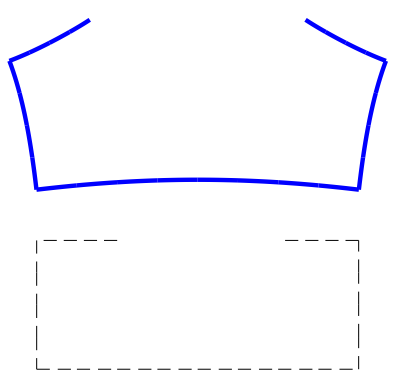

a) All modes

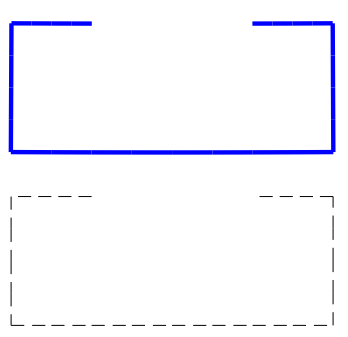

b) All polynomial modes

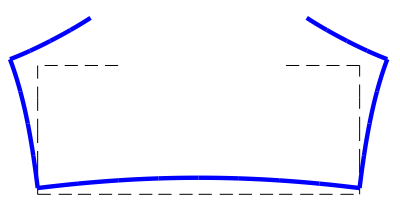

c) All exponential modes

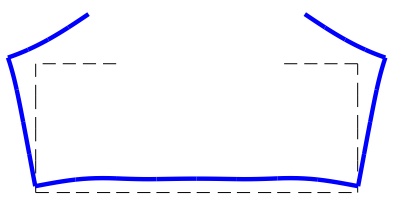

d) Selected exponential modes

Figure 21: Ex. 4 - The transverse cross-sectional deformation at the point where the loads are acting (this is at $Z=1000 \mathrm{~mm})$. The deformations are scaled 20 times

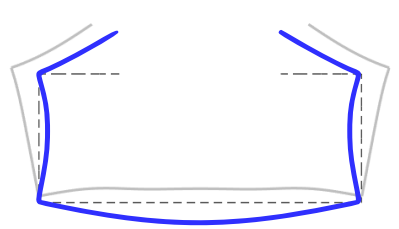

a) Mode 23

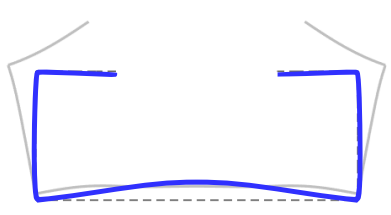

d) Mode $31+32$

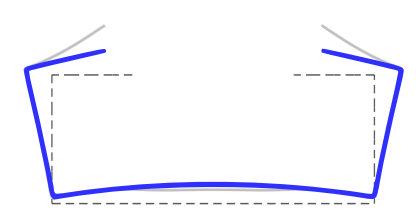

b) Mode $15+16$

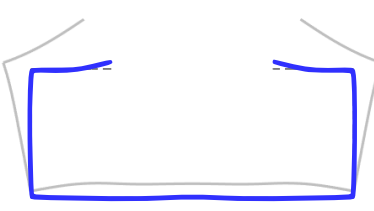

e) Mode 53

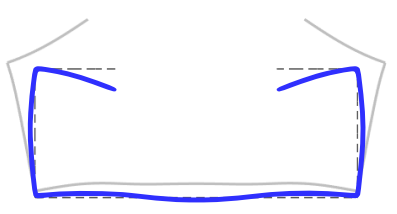

c) Mode $45+46$

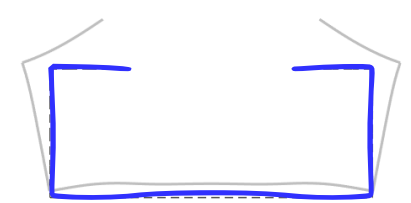

f) Remaining modes

Figure 22: Ex. 4 - Transverse exponential displacement modes at $Z=1000 \mathrm{~mm}$. The light grey cross-section is the one from Figure 21 . The displacement modes are scaled by a factor of 20

only include a limited number of degrees of freedom compared to, for example, a similar analysis using finite shell elements in the framework of the finite element method. Again, this is a result of the exact beam interpolation functions. Hence, the derivation of these axial interpolation functions is a result of the procedure deducing the beam element stiffness matrix, which is based on the novel semianalytic cross-section analysis presented by the authors in [31. Straight wall elements is used to discretise the thinwalled cross-section centre line, and with a strain energy formulation, a system of cross-sectional equilibrium equations is deduced. This formulation is purely based on linear elastic constitutive relations and isotropic material prop- erties. The system of equilibrium equations is solved as a polynomial eigenvalue problem, and cross-sectional displacement fields are computed as eigenvectors with the associated eigenvalues being related to axial amplitude functions. The derivation of beam displacement modes is decomposed into two parts. One is related to fundamental beam modes and the other to distortional beam modes. The former part of the modes is belonging to the zero eigenvalues, which is found through a systematic decomposition and combination of the related eigenvectors. This is a procedure which is used by Morandini et al. [42] and Genoese et al. [25, 26]. The latter part of the modes is based on the non-zero eigensolutions. 

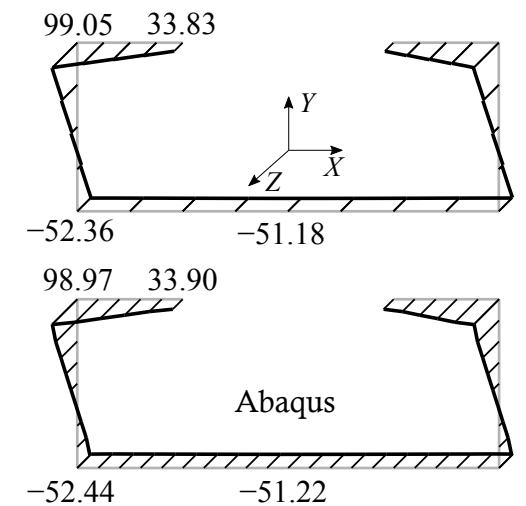

Figure 23: Ex. $4-$ Axial stresses, $\sigma_{z z}$, in $[\mathrm{MPa}]$ at $Z=950 \mathrm{~mm}$ and $n=0 \mathrm{~mm}$

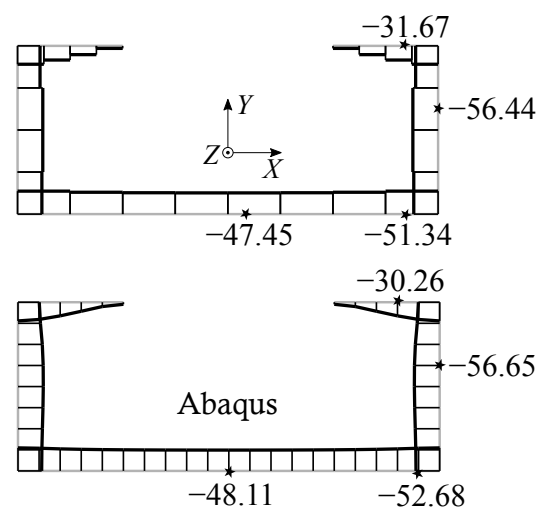

Figure 24: Ex. 4 - Transverse bending stresses, $\sigma_{s s}$, in $[\mathrm{MPa}]$ at $Z=950 \mathrm{~mm}$ and $n=t / 2$. The star indicates to which element or node a given stress corresponds

Through examples, usability of the beam element is illustrated and reasonable agreement with results obtained with a commercial finite element program is achieved. However, near corners, in the cross-section, local shear effects occur due to the transmission of shear stresses around corners. This leads to a shear stress distribution that deviates from those shear stresses obtained in more classic shell models obeying the Kirchhoff plate hypothesis. Nonetheless, considering detailed solid finite element models, a similar transmission of shear stresses is seen confirming the results obtained in this theory. As a consequence, this theory results in a detailed assessment of shear stress transmission between non-aligned wall elements. However, a fine discretisation with wall elements is to be preferred close to these regions to get improved results. This is due to the attenuation of the shear stresses being no more than twice the wall thickness.

It has been shown in one of the examples how deformation of a cross-section can be decomposed into displacement modes. This mode decomposition is directly found from the solutions as the displacement mode intensity factors, which indicates the influence of each mode. Thus, indepth knowledge of the beam deformation can be obtained right away and is an integrated part of the solution. This is one of the main advantages of this theory in comparison with for example cFSM where constraint equations are to be added to the kinematic equations to obtain a similar mode decomposition.

During a mode decomposition, the attenuation of the exponential distortion modes can be found since this is directly related to the eigenvalues, which were deduced computing the cross-sectional beam displacement modes. This is exemplified and verified in one of the examples. Furthermore, this mode decomposition may be utilised in future formulations of efficient beam elements with a reduced number of modal degrees of freedom based on a subset of modes. Besides, the identification of displacement modes becomes essential extending the theory to include buckling and post-buckling analysis of the beam members as well. Indeed, higher-order exponential displacement modes play a crucial role here, which is also highlighted in [27.

\section{Acknowledgement}

The first author wishes to thank the Danish consultancy company NIRAS A/S and the Innovation Fund Denmark, grant 5189-00005B, for funding this project.

\section{References}

[1] K. E. Kurrer, History of the Theory of Structures: From Arch Analysis To Computational Mechanics, John Wiley \& Sons, 2009 (2009). doi:10.1002/9783433600160

[2] V. Z. Vlasov, Thin-Walled Elastic Beams, 2nd Edition, Jerusalem, 1961, israel Program for scientific translations (1961).

[3] C. F. Kollbrunner, N. Hajdin, Dünnwandige Stäbe · Band 1, Stäbe mit undeformierbaren Querschnitten, Springer-Verlag, 1972 (1972).

[4] D. Krajcinovic, A consistent discete elements technique for thinwalled assemblages, International Journal of Solids and Structures 5 (1969) 639-662 (1969). doi:10.1016/0020-7683(69) 90085-7.

[5] R. S. Barsoum, R. H. Gallagher, Finite element analysis of torsional and torsional-flexural stability problems, International Journal for Numerical Methods in Engineering 2 (1970) 335352 (1970). doi:10.1002/nme.1620020304

[6] R. Schardt, Eine erweiterung der technishen biegelehre für die berechnung biegestreifer prismatischer falfwerke, Der Stahlbau 35 (1966) 161-171 (1966).

[7] R. Schardt, Verallgemeinerte Technische Biegetheorie - Band 1, Lineare Theorie, 2nd Edition, Metrum-Verlag,Darmstadt, 1989 (1989).

[8] C. F. Kollbrunner, N. Hajdin, Dünnwandige Stäbe · Band 2, Stäbe mit deformierbaren Querschnitten - Nicht-elastiches Verhalten dünnwandiger Stäbe, Springer-Verlag, 1975 (1975).

[9] J. M. Davies, P. Leach, First-order generalised beam theory, Journal of Constructional Steel Research 31 (1994) 187-220 (1994). doi:10.1016/0143-974X(94)90010-8

[10] J. M. Davies, P. Leach, D. Heinz, Second-order generalised beam theory, Journal of Constructional Steel Research 31 (1994) 221-241 (1994). doi:10.1016/0143-974X(94)90011-6.

[11] P. Simão, L. S. da Silva, A unified energy formulation for the stability analysis of open and closed thin-walled members in the framework of the generalized beam theory, Thin-Walled Structures 42 (2004) 1495-1517 (2004). doi:10.1016/j.tws.2004. 03.021 
[12] N. Silvestre, D. Camotim, First-order generalised beam theory for arbitrary orthotropic materials, Thin-Walled Structures 40 (2002) 755-789 (2002). doi:10.1016/S0263-8231(02)00025-3

[13] N. Silvestre, D. Camotim, Second-order generalised beam theory for arbitrary orthotropic materials, Thin-Walled Structures 40 (2002) 791-820 (2002). doi:10.1016/S0263-8231(02) 00026-5.

[14] N. Silvestre, D. Camotim, Nonlinear generalized beam theory for cold-formed steel members, International Journal of Structural Stability and Dynamics 3 (4) (2003) 461-490 (2003). doi:10.1142/S0219455403001002

[15] N. Silvestre, D. Camotim, N. F. Silva, Generalized beam theory revisited: from kinematical assumptions to the deformation mode determination, International Journal of Structural Stability and Dynamics 11 (2011) 969-997 (2011). doi:10.1142/ S0219455411004427

16] D. Camotim, C. Basaglia, Buckling Analysis of Thin-Walled Structures Using Generalized Beam Theory (GBT): A Stateof-the-art Report, Steel Construction 6 (2013) 117-131 (2013). doi:10.1002/stco.201310021

[17] C. Basaglia, D. Camotim, N. Silvestre, Global Buckling Analysis of Plane and Space Thin-Walled Frames in the Context of GBT, Thin-Walled Structures 46 (2008) 79-101 (2008). doi: 10.1016/j.tws.2007.07.007

[18] G. Taig, G. Ranzi, Generalised beam theory (gbt) for stiffened sections, International Journal of Steel Structures 14 (2014) 381-397 (2014). doi:10.1007/s13296-014-2017-x

19] G. Taig, G. Ranzi, A. Luongo, Gbt pre-buckling and buckling analyses of thin-walled members under axial and transverse loads, Continuum Mechanics and Thermodynamics 28 (2016) 41-66 (2016). doi:10.1007/s00161-014-0399-9

[20] Y. K. Cheung, Finite Strip Method in Structural Analysis, Pergamon Press, Oxford, New York, Toronto, Sydney, Paris, Frankfurt, 1976 (1976).

[21] S. Ádány, B. W. Schafer, Buckling mode decomposition of single-branched open cross-section members via finite strip method: Derivation, Thin-Walled Structures 44 (2006) 563-584 (2006). doi:10.1016/j.tws.2006.03.013

[22] S. Ádány, B. W. Schafer, Buckling mode decomposition of single-branched open cross-section members via finite strip method: Application and examples, Thin-Walled Structures 44 (2006) 585-600 (2006). doi:10.1016/j.tws.2006.03.014

[23] S. Ádány, B. W. Schafer, A full modal decomposition of thinwalled, single-branched open cross-section members via the constrained finite strip method, Journal of Constructional Steel Research 64 (1) (2008) 12-29 (2008). doi:10.1016/j.jcsr.2007. 04.004

[24] S. Ádány, N. Silvestre, B. W. Schafer, D. Camotim, GBT and cFSM: Two modal approaches to the buckling analysis of unbranched thin-walled members, Advanced Steel Construction 5 (2) (2009) 195-223 (2009).

[25] A. Genoese, A. Genoese, A. Bilotta, G. Garcea, A generalized model for heterogeneous and anisotropic beams including section distortions, Thin-Walled Structures 74 (2014) 85-103 (2014). doi:10.1016/j.tws.2013.09.019

[26] A. Genoese, A. Genoese, A. Bilotta, G. Garcea, Buckling analysis through a generalized beam model including section distortion, Thin-Walled Structures 85 (2014) 125-141 (2014). doi:10.1016/j.tws.2014.08.012

27] G. Garcea, R. Gonçalves, A. Billota, D. Manta, R. Bebiano, N. Leonetti, D. Magisano, D. Camotim, Deformation modes of thin-walled members: a comparison between the method of generalized eigenvectors and generalized beam theory, ThinWalled Structures 100 (2016) 192-212 (2016). doi:10.1016/ j.tws.2015.11.013

[28] J. Jönsson, M. J. Andreassen, Distortional eigenmodes and homogeneous solutions for semi-discretized thin-walled beams, Thin-Walled Structures 49 (2011) 691-707 (2011). doi:10. 1016/j.tws.2010.12.009

[29] R. F. Vieira, F. B. E. Virtuoso, E. B. R. Pereira, A higher order thin-walled model including warping and shear modes, Inter- national Journal of Mechanical Sciences (2013) 67-82 (2013). doi:10.1016/j.ijmecsci.2012.10.009

30] R. F. Vieira, F. B. Virtuoso, E. B. R. Pereira, A higher order model for thin-walled structures with deformable cross-sections, International Journal of Solids and Structures (2014) 575-598 (2014). doi:10.1016/j.ijsolstr.2013.10.023

[31] A. B. Hansen, J. Jönsson, Displacement modes of a thin-walled beam model with deformable cross section, Thin-Walled Structures 141 (2019) 576-592 (2019). doi:10.1016/j.tws.2019.01. 052

[32] R. F. Vieira, A higher order thin-walled beam model, Ph.D. thesis, Universidade Técnica de Lisboa - Instituto Superior Técnico (2010)

[33] A. B. Hansen, J. Jönsson, A gbt-framework towards modal modelling of steel structures, John Wiley \& Sons, Ltd - ce/papers (2017) 1822-1830 (2017). doi:10.1002/cepa.226

34] S. P. Timoshenko, LXVI. On the Correction for Shear of the Differential Equation for Transverse Vibrations of Prismatic Bars, Philosophical magazine series 641 (245) (1921) 744-746 (1921). doi: $10.1080 / 14786442108636264$

35] G. R. Cowper, The shear coefficient in timoshenko's beam theory, Journal of Applied Mechanics (1966) 335-340 (1966). doi:10.1115/1.3625046

[36] R. D. Cook, D. S. Malkus, M. E. Plesha, Concepts and applications of finite element analysis, 3rd Edition, John Wiley \& Son, NewYork Chichester Brisbane Toronto Singapore, 1989 (1989).

[37] F. Tisseur, K. Meerbergen, The quadratic eigenvalue problem, Society for Industrial and Applied Mathematics 43 (2) (2001) 235-286 (2001). doi:10.1.1.32.9042

[38] M. J. Andreassen, J. Jönsson, Distortional solutions for loaded semi-discretized thin-walled beams, Thin-Walled Structures 50 (2012) 116-127 (2012). doi:10.1016/j.tws.2011.08.013.

[39] J. Jönsson, Distortional theory of thin-walled beams, ThinWalled Structures 33 (1999) 269-303 (1999). doi:10.1016/ S0263-8231(98) 00050-0

[40] V. Giavotto, M. Borri, P. Mantegazza, G. Ghiringhelli, Anisotropic beam theory and applications, Computers \& Structures 16 (1983) 403-413 (1983). doi:10.1016/0045-7949(83) 90179-7.

[41] Abaqus, Abaqus/CAE 2016; Abaqus ${ }^{\circledR}$ and SIMULIA ${ }^{\circledR}$ used for finite element analysis, 2016, abaqus Inc., SIMULIA @ D Dassault Systèmes, 2015, Version 2016 (2016).

[42] M. Morandini, M. Chierichetti, P. Mantegazza, Characteristic behavior of prismatic anisotropic beam via generalized eigenvectors, International Journal of Solids and Structures 47 (2010) 1327-1337 (2010). doi:10.1016/j.ijsolstr.2010.01.017 